\title{
Slug Self-Propulsion in a Capillary Tube Mathematical Modeling and Numerical Simulation
}

\author{
M. I. Khodabocus, ${ }^{1}$ M. Sellier, ${ }^{1}$ and V. Nock $^{2}$ \\ ${ }^{1}$ Department of Mechanical Engineering, University of Canterbury, Private Bag 4800, Christchurch 8140, New Zealand \\ ${ }^{2}$ Department of Electrical and Computer Engineering, University of Canterbury, Private Bag 4800, \\ Christchurch 8140, New Zealand
}

Correspondence should be addressed to M. Sellier; mathieu.sellier@canterbury.ac.nz

Received 1 February 2016; Accepted 20 June 2016

Academic Editor: Xavier Leoncini

Copyright (C) 2016 M. I. Khodabocus et al. This is an open access article distributed under the Creative Commons Attribution License, which permits unrestricted use, distribution, and reproduction in any medium, provided the original work is properly cited.

\begin{abstract}
A composite droplet made of two miscible fluids in a narrow tube generally moves under the action of capillarity until complete mixture is attained. This physical situation is analysed here on a combined theoretical and numerical analysis. The mathematical framework consists of the two-phase flow phase-field equation set, an advection-diffusion chemical concentration equation, and closure relationships relating the surface tensions to the chemical concentration. The numerical framework is composed of the COMSOL Laminar two-phase flow phase-field method coupled with an advection-diffusion chemical concentration equation. Through transient studies, we show that the penetrating length of the bidroplet system into the capillary tube is linear at early-time regime and exponential at late-time regime. Through parametric studies, we show that the rate of penetration of the bidroplet system into the capillary tube is proportional to a time-dependent exponential function. We also show that this speed obeys the Poiseuille law at the early-time regime. A series of position, speed-versus-property graphs are included to support the analysis. Finally, the overall results are contrasted with available experimental data, grouped together to settle a general mathematical description of the phenomenon, and explained and concluded on this basis.
\end{abstract}

\section{Introduction}

When two physically distinct but miscible liquid droplets are placed in a capillary tube, a key feature that is commonly observed is the bulk motion of the composite droplet under the action of capillarity until complete mixing is attained [1]. In Figure 1 is given a freehand sketch of this scenario. This is an example of motion due to (chemical) Marangoni effect [2-4]. The motion of the bidroplet system is caused by local variations of its advancing and receding surface tensions that are in turn caused by differences in its chemical compositions. Out of this simple scenario grew two kinematical concepts, namely, the penetrating length and the penetrating rate. When both concepts are grouped together, we attain, by inference, slug self-propulsion mechanism-a juxtaposition of two miscible liquid droplets. An important facet of this driving mechanism is that it is simple and so could be delicately adjusted to manipulate liquid droplets in small-scale fluidic devices. Earlier scientists have studied the phenomenon experimentally and have concluded that it might be used as a means of self-propelling, transporting microscale substances at a respectable penetrating length and rate, respectively, of orders of centimeters, centimeters per second $[1,5,6]$.

Bouasse [7] inquired experimentally on capillarity and interfacial phenomena. He found that a droplet predeposited in a conic tube undergoes motion towards the narrow region because of the Laplacian pressure difference induced on both its menisci. Weislogel [8] investigated the dynamics of a liquid droplet in a chemically asymmetric tube, by treating the tube to be successively hydrophilic and hydrophobic. He found the droplet to move towards the hydrophilic side. Dos Santos and Ondarçuhu [9] argued that a droplet containing a reactive substance remained in its state of motion as long as such reactive agent is operative-the dynamics of reactive wetting on solid surfaces were discussed by De Gennes [10]. 
Slug self-propulsion in a capillary tube

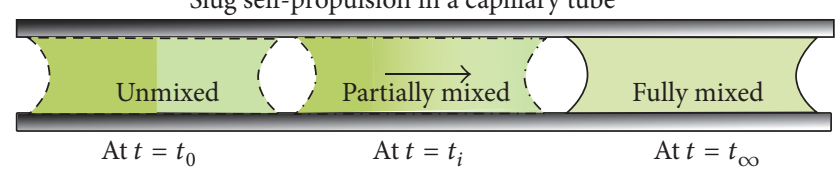

FIGURE 1: Illustration of the slug self-propulsion phenomenon in a capillary tube.

Historically, it may be that the first documented study goes back to 1712, when Hauksbee [11] investigated the behaviour of a drop of oil of oranges sandwiched in between two nonparallel glass plates. In that paper, two points obviously follow in the light of this phenomenon. First, the fact that the motion of the drop reversed when the slope of those two plates was reversed led Hauksbee to believe that the direction of motion is towards the narrow region. Secondly, the fact that his experiment was reproducible in vacuum further informed Hauksbee that such motion is solely due to interfacial phenomenon.

Currently, existing (active) techniques of manipulating discrete droplets include electrowetting [12-17], dielectrophoresis [18], thermocapillary [19-22], surface acoustic wave (SAW) [23, 24], and pressure-driven based-channel systems [25]. Thanks to these sophisticated techniques, the fields of nano- and microfluidics have seen over the past decade considerable development.

Self-propulsion phenomena as well as coalescence phenomena are found to have generated considerable interest and many features have been correctly described and explained as well. Some fundamental questions were addressed by De Gennes [10, 26, 27] and Shanahan and De Gennes [28]. Shanahan and De Gennes stressed that the motion of the system is switched on mainly due to the slight perturbation communicated to the system at the time when the second droplet is brought into close contact to the predeposited one. Furthermore, the authors pointed out that for motion to occur the system must necessarily be asymmetric and that such motion is maintained only insofar that such character is observed in the system.

Recently, there has been a growing interest in exploiting droplets self-propulsion phenomenon, thereby avoiding the arduous fabrication procedures required $[6,29,30]$. For instance, Karpitschka and Riegler [31] gave an account on the coalescence of liquid droplets in two respective regimes, namely, the fast coalescence or delayed coalescence. In the paper of Karpitschka and Riegler, the authors pointed out that the coalescence phenomenon is predominantly governed by surface tension, regardless of whether it is fast or delayed coalescence. For instance, when the difference $\delta \gamma$ in the surface tensions of the working droplets is such that $\delta \gamma \approx$ $3 \mathrm{mN} \cdot \mathrm{m}^{-1}$, delayed coalescence phenomenon is observed from the bidroplet system and, for all other cases such that $\delta \gamma<3 \mathrm{mN} \cdot \mathrm{m}^{-1}$, fast coalescence phenomenon is observed. In a subsequent paper, Karpitschka and Riegler [32] explore the study of delayed droplets coalescence phenomenon on a combined experimental and theoretical basis.
On the one hand, the self-propulsion of liquid droplets has also been addressed by Sinz and Darhuber [6] and Sellier et al. [29, 30]. In the works of Sellier et al., the authors examined the self-propulsion mechanisms in unconfined systems, that is, sessile droplets configuration. The principles of manipulating discrete droplets using active methods were considered by Darhuber, Valentino, Davis, and Troian $[33,34]$. In the paper of Sinz and Darhuber, the authors addressed the self-propelling of surfactant droplets in chemically confined systems. Therein, Sinz and Darhuber argued that this passive driving mechanism might be used to transport microscale substances.

Lunati and Or [35] undertook the problem of gravitydriven slug dynamics in capillary tubes. The authors have answered questions regarding the origin, strength, and effects of the drag-forces developed at the contact lines. They also studied the kinematics of the slug, contrasting the numerical simulation results with the experiment results of Bico and Quéré [36] on falling of viscous slugs in vertical capillary tubes. Bico and Quéré deduced a set of scaling relationships characterising the steady motion of the slug. On the experimental side, the earliest laboratory experiments wherein the subject matter based on purely confined systems has been explored thoroughly were those of Bico and Quéré $[1,5]$. These are the most important works in close connection with the subject at hand. In these, more work was endeavoured on the dynamics of trains of droplets in horizontal capillary tubes in both wetting and drying conditions. For instance, in the former paper of Bico and Quéré [5], which constitute their preliminary ground-based experiments, they argued mainly on the coating properties of trains of juxtaposed liquid droplets in terms of geometrical and viscous effects-another very interesting field of inquiries; they moreover drew attention on their versatility towards integrated microfluidic devices. For example, Bico and Quéré [1] have studied the response of the system in more complex geometries and displayed more features on a combined experimental and theoretical basis.

Since then, only few scientists have begun to study the subject on theoretical and numerical sides. Furthermore, studies numerically based on the slug self-propulsion phenomenon do not appear to have received much attention. To sum up, it may be concluded that we found no scientist has come to ponder on the self-propelling phenomenon in confined system quantitatively and to arrive at a more rational conception of it.

We have studied carefully the dynamics of a two miscible fluids in a capillary tube and, accordingly, found the law describing the position (or penetrating length) of the slug into the capillary tube is well described by the following relationship: $X_{s}(t) \approx \eta_{1}\left\{1-\exp \left(-\eta_{2}\left(t-t_{0}\right)\right)\right\}$, where $\eta_{1}$, $\eta_{2}>0$ are property-dependent constants. Furthermore, it is observed at the early-time that the slug velocity (or rate of penetration), that is, $U_{s}=\partial_{t} X_{s}(t)$, obeys a Poiseuille law: $U_{s} \propto$ $R\left(\gamma_{1,0}-\gamma_{2,0}\right) / 4 \mu l_{s}$. In this expression, $R$ designates the radius of the capillary tube; $\gamma_{1,0}, \gamma_{2,0}>0$ are the surface tensions of the miscible fluids; and $\mu, l_{s}>0$ are the dynamic viscosity and length of the slug. We came to these laws numerically by collecting data on the slug position as a function time and 


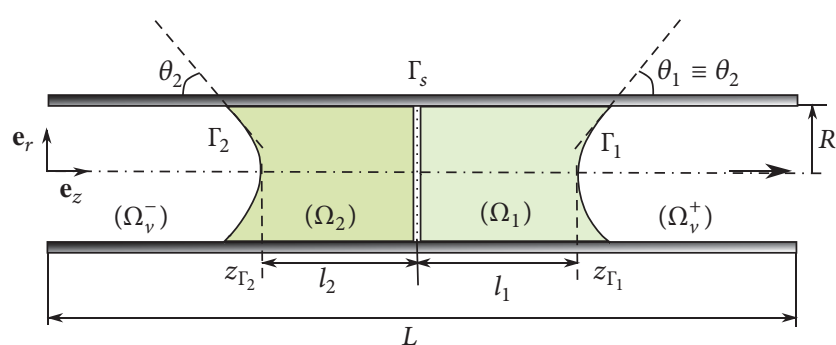

FIGURE 2: Illustration of a slug composed of two miscible fluids $\Omega_{1} \cup$ $\Omega_{2}$, both of which bounded partly by the capillary tube $\Gamma_{s}$ and by $\Gamma_{k}$ (with $k=1,2$ ) by a still vapour phase $\Omega_{v}^{ \pm}$. The lengths $L, l_{1}, l_{2}>0$ denote the length of the capillary tube and droplets 1 and 2 . Bold arrow denotes the direction of its motion.

the maximum travelled distance. An inspection of the form of the property $U_{s}$ (discussed hereinafter) evidences at once that small and low viscous substances are speedier than large and highly viscous ones.

In this paper, an account of the mathematical development is given. The paper is organised as follows: Section 2 is devoted to mathematical formulations and Section 3 to the numerical implementation of the mathematical framework. Results from numerical experiments and analysis are presented in Section 4: the motions of the slug with respect to time and property constants are discussed in Sections 6 and 7, respectively; the results are contrasted with the papers of Bico and Quéré $[1,5]$. In Section 8, a power-law model describing the position of the slug with respect to time and the leading property constants involved is established. The overall results are finally argued in Section 9.

\section{Mathematical Formulations}

2.1. Preliminaries. First, some aspects, definitions, and notations are in order. Truly speaking, we examine here a threefluid flow problem, the properties of which are such that two of which are miscible phases but immiscible with the third one. In this context, their respective domains of definition are written as

$$
\begin{aligned}
& \Omega_{v}^{-} \cup \Omega_{v}^{+} \stackrel{\text { def }}{=}\left\{(r, z): 0 \leq r \leq R, \quad\left(0 \leq z<z_{\Gamma_{2}}\right)\right. \\
& \left.\cup\left(z_{\Gamma_{1}}<z \leq L\right)\right\}, \\
& \Omega_{1} \cup \Omega_{2} \stackrel{\text { def }}{=}\left\{(r, z): 0 \leq r \leq R, z_{\Gamma_{2}} \leq z \leq z_{\Gamma_{1}}\right\},
\end{aligned}
$$

where $\mathbf{r}=(r, z)^{T}$ is a point of either the set $\Omega_{v}^{-} \cup \Omega_{v}^{+}$or $\Omega_{1} \cup \Omega_{2}$. The scalar fields $z_{\Gamma_{i}}(t)$ (with $i=1,2$ ) are the respective time-dependent positions of the (front and rear) interfaces of the slug. Obviously, the sets $\Gamma_{1}=\Omega_{1} \cup \Omega_{v}^{+}$and $\Gamma_{2}=\Omega_{1} \cup \Omega_{v}^{-}$denote those time-dependent interfaces. These physical domains, sets (1), are clearly illustrated in Figure 2. The choice of Figure 2 is motivated by that of Figure 1. In view of the foregoing definitions, it thus appears evident to regard $\Omega_{1} \cup \Omega_{2} \subset \mathbb{R}^{2}$ merely as a two-miscible-liquid region and $\Omega_{v}^{ \pm}=\Omega_{v}^{-} \cup \Omega_{v}^{+} \subset \mathbb{R}^{2}$ the (disjoint) union of its rear and front vapour phase regions. In fact, with these underlying definitions in mind, both phases can be related without loss of generality as thus

$$
\begin{aligned}
& \operatorname{vol}\left(\Omega_{v}^{-} \cup \Omega_{1} \cup \Omega_{2} \cup \Omega_{v}^{+}\right) \\
& =\operatorname{vol}\left(\Omega_{1} \cup \Omega_{2}\right)+\operatorname{vol}\left(\Omega_{v}^{-} \cup \Omega_{v}^{+}\right),
\end{aligned}
$$

where $\operatorname{vol}(\cdot)$ denotes the volume of its arguments. Furthermore, we characterise by the scalar fields $\phi_{12}, \phi_{v} \in[0,1]$ the functionals describing the volume fraction of fluids in $\Omega_{1} \cup \Omega_{2} \subset \mathbb{R}^{2}$ and $\Omega_{v}^{-} \cup \Omega_{v}^{+} \subset \mathbb{R}^{2}$, respectively. Consequently, by virtue of the above postulation, (2), it results in

$$
\begin{aligned}
1= & \frac{\operatorname{vol}\left(\Omega_{1} \cup \Omega_{2}\right)}{\operatorname{vol}\left(\Omega_{v}^{-} \cup \Omega_{1} \cup \Omega_{2} \cup \Omega_{v}^{+}\right)} \\
& +\frac{\operatorname{vol}\left(\Omega_{v}^{-} \cup \Omega_{v}^{+}\right)}{\operatorname{vol}\left(\Omega_{v}^{-} \cup \Omega_{1} \cup \Omega_{2} \cup \Omega_{v}^{+}\right)}=\phi_{12}+\phi_{v},
\end{aligned}
$$

where $\left.\left.\phi_{12}\right\rfloor_{\Gamma_{i}} \simeq \phi_{v}\right\rfloor_{\Gamma_{i}} \simeq 1 / 2$ (with $\left.i=1,2\right)$. In regard to these facts, the problem under consideration now reads an Immiscible Two-Phase Flow problem. At this point, we have made the essential geometrical features prominent and, in what follows, a two-phase flow concentration-dependent surface tension problem of a viscous liquid slug in a capillary tube is undertaken.

\subsection{Transport of Concentration by Diffusion and Convection.} Denote by the scalar field $\mathscr{C}^{(l)}(\mathbf{r}, t)$ the chemical concentration of a (highly) volatile substance filling the region $\Omega_{2}$ initially (note that the other substance filling the region $\Omega_{1}$ is taken to be lowly volatile). To establish the transport equation for the chemical concentration function $\mathscr{C}^{(l)}(\mathbf{r}, t)$ in $\Omega_{1} \cup \Omega_{2}$, we reason in the following way.

Initially, postulate the concentration within the slug to be such that

$$
\mathscr{C}^{(l)}(\mathbf{r}, t)=i-1 \quad \text { in } \Omega_{i} \times\{t=0\} \quad(i=1,2) ;
$$

and presuppose a normally imposed flux $\varphi_{i} \geq 0$ on the corresponding boundary $\Gamma_{i}$ (with $i=1,2, s$ ) by its external surroundings. Then, by virtue of these assertions, $\mathscr{C}^{(l)}(\mathbf{r}, t)$ is simply described by the equation of chemical species transported by diffusion and convection (CD-model, say), as

$$
\begin{aligned}
& \partial_{t} \mathscr{C}^{(l)}+\operatorname{div}\left(\mathscr{C}^{(l)} \mathbf{u}\right)-\operatorname{div}\left(D_{21} \nabla \mathscr{C}^{(l)}\right)=0 \\
& \text { in } \bigcup_{i=1,2} \Omega_{i} \times(0, T) ; \\
&-\mathbf{n}_{i} \cdot\left(\mathscr{C}^{(l)} \mathbf{u}-D_{21} \nabla \mathscr{C}^{(l)}\right)=\varphi_{i} \\
& \text { on } \Gamma_{i} \times(0, T) \quad(i=1,2, s) ; \\
& \mathscr{C}^{(l)}(\mathbf{r}, t)=i-1 \\
& \text { in } \Omega_{i} \times\{0\} \quad(i=1,2) ;
\end{aligned}
$$

for given $T>0$. In (5), the proportionality factor $D_{21}>$ 0 is the diffusivity of fluid 1 into 2 ; the vector field $\mathbf{n}_{i}(\mathbf{r}, t)$ 
denotes the outward normal unit vector corresponding to the boundary $\Gamma_{i}$ (with $i=1,2, s$ ). It may be pointed out in passing that this convection-diffusion model, that is, (5), merits further attention, for it should also agree with the subsequent changes in position of the slug. This point is clarified in a later section.

2.3. Inclusion of Concentration-Dependent Surface Tension. We now discuss proper forms of interfacial forces at the boundaries $\Gamma_{1}, \Gamma_{2}$. Supposing a solution for the scalar field $\mathscr{C}^{(l)}(\mathbf{r}, t)$ is granted, we can then define $\gamma_{i}\left(\mathscr{C}_{\Gamma_{i}}^{(l)}\right)$ as the growth, $i=1$ (resp., decay, $i=2$ ) of interfacial effects in terms of $\left.\mathscr{C}_{\Gamma_{i}}^{(l)}=\mathscr{C}^{(l)}\right\rfloor_{\Gamma_{i}}$ at their respective boundaries $\Gamma_{1}, \Gamma_{2}$. In order to deduce such proper forms for $\gamma_{i}\left(\mathscr{C}_{\Gamma_{i}}^{(l)}\right)$, we shall in the first place consider the following facts: initially, the domains $\Omega_{1}$, $\Omega_{2}$ are characterised to be such that $\mathscr{C}^{(l)}=0$ in $\Omega_{1}$ and $\mathscr{C}^{(l)}=$ 1 in $\Omega_{2}$, and, finally, both are expected to be in equilibrium (chemically), attaining the value $\mathscr{C}_{\infty}^{(l)} \in(0,1)$, say; thence, the correct conditions to be imposed upon $\mathscr{C}_{\Gamma_{k}}^{(l)}(\mathbf{r}, t)$ (with $i=1$, 2) simply write as

$$
\begin{aligned}
& \mathscr{C}_{\Gamma_{i}}^{(l)}=i-1 \quad \text { on } \Gamma_{i} \times\{t=0\} \quad(i=1,2) ; \\
& \mathscr{C}_{\Gamma_{i}}^{(l)}=\mathscr{C}_{\infty}^{(l)} \text { on } \bigcup_{i=1,2} \Gamma_{i} \times\{t \longrightarrow \infty\} .
\end{aligned}
$$

Taking (6) into consideration along with an additional assertion that the surface tensions evolve linearly, one arrives at

$$
\begin{aligned}
\gamma_{i}\left(\mathscr{C}_{\Gamma_{i}}^{(l)}\right)=\frac{\mathscr{C}_{\infty}^{(l)}-\mathscr{C}_{\Gamma_{i}}^{(l)}}{\mathscr{C}_{\infty}^{(l)}-(i-1)} \gamma_{i, 0} & +\frac{\mathscr{C}_{\Gamma_{i}}^{(l)}-(i-1)}{\mathscr{C}_{\infty}^{(l)}-(i-1)} \gamma_{i, \infty} \\
& \text { on } \Gamma_{i} \times(0, T)(i=1,2) .
\end{aligned}
$$

The linearity of $\gamma_{i}\left(\mathscr{C}_{\Gamma_{i}}^{(l)}\right)$ (with $\left.i=1,2\right)$ on the scalar field $\mathscr{C}^{(l)}(\mathbf{r}, t)$ is here motivated by the works of Carles and Cazabat $[37,38]$ on the spreading dynamics of Newtonian droplets. Therein, Carles and Cazabat [37] found that the surface tension of those mixed substances varied roughly linearly with the chemical concentration of the mixture. Equation (7) is physically reasonable, for $\mathscr{C}^{(l)} \in\left[0, \mathscr{C}_{\infty}^{(l)}\right]$ in $\Omega_{1}$ and $\mathscr{C}^{(l)} \in\left[\mathscr{C}_{\infty}^{(l)}, 1\right]$ in $\Omega_{2}$. However, out of (7) grew one important question which is somehow unclear.

Question 1. What are the magnitudes of $\mathscr{C}_{\infty}^{(l)}, \gamma_{i, \infty}>0$ (with $i=1,2)$ ?

The answer of this question is found in the following line of reasoning. First, it is evident that

$$
\begin{aligned}
& \lim _{l_{2} \rightarrow 0} \mathscr{C}_{\infty}^{(l)}=0, \\
& \lim _{l_{2} \rightarrow \infty} \mathscr{C}_{\infty}^{(l)}=1
\end{aligned}
$$

where the geometrical property $l_{1}>0$ stands for the size of $\Omega_{1}$ along the $z$-axis (see Figure 2). That is, the (chemical) equilibrium state is somewhat dependent upon the geometrical properties of the slug, as found in the paper of Bico and Quéré [1]. Thus, the expression which would match these conditions is $\mathscr{C}_{\infty}^{(l)}=l_{2} /\left(l_{1}+l_{2}\right)$, since $\mathscr{C}^{(l)}=i-1$ in $\Omega_{i}$ (with $\left.i=1,2\right)$ at time $t=0$. Note that $\mathscr{C}_{\infty}^{(l)}=1 / 2$ is also an admissible value but only for a particular case so to speak when $l_{1}=l_{2}$, for it neglects the geometrical property of the slug, particularly, when $l_{2} \rightarrow 0$, $\infty$.

In regard to these arguments, one then concludes that the lesser the length $l_{2}>0$ the higher the concentration $\mathscr{C}_{\infty}^{(l)} \in$ $(0,1)$. It now remains to settle proper forms for $\gamma_{i, \infty}>0$ (with $i=1,2)$. First, we note that

$$
\begin{gathered}
\lim _{t \rightarrow \infty} \frac{l_{1} \gamma_{1}+l_{2} \gamma_{2}}{l_{1}+l_{2}}=\frac{l_{1} \gamma_{1, \infty}+l_{2} \gamma_{2, \infty}}{l_{1}+l_{2}}=\frac{l_{1} \gamma_{1,0}+l_{2} \gamma_{2,0}}{l_{1}+l_{2}} \\
\text { on } \bigcup_{i=1,2} \Gamma_{i} ; \\
\gamma_{2-i, \infty} \equiv \gamma_{2-i, 0} \quad \text { when } l_{1+i}=0(i=0,1) .
\end{gathered}
$$

Hence, these suggest to consider a barycentric-based model, namely, of the form

$$
\gamma_{i, \infty}=\frac{l_{1} \gamma_{1,0}+l_{2} \gamma_{2,0}}{l_{1}+l_{2}}, \quad \text { on } \Gamma_{i}(i=1,2) \text {, }
$$

because $\gamma_{1, \infty}=\gamma_{2, \infty}$ (equilibrium surface tension) when $t \rightarrow \infty$. Clearly, there is no loss of generality in doing so, for not only does (10) agree well but it is also a physically acceptable expression. We are now well prepared to set forth the governing equations; this is the purpose of the next section.

2.4. Two-Phase Flow Phase-Field Model. In Sections 2.2 and 2.3 , we covered much ground on diffusive and interfacial effects. The present section takes those effects into account and models the slug self-propulsion mechanism using the phase-field approach. In a sense, we have beforehand the motion of a two-miscible-liquid system partly in contact with an immiscible one, the vapour phase region. Therefore, the starting point is naturally based on the classical NavierStokes equations (NS-model, say) for incompressible flow with interfacial forces coupled with the so-called CahnHilliard evolution equation ( $\mathrm{CH}$-model, say). In the absence of gravitational field, those equations in vector invariant forms read as follows:

$$
\begin{gathered}
\partial_{t}(\rho \mathbf{u})+\operatorname{div}(\rho \mathbf{u} \otimes \mathbf{u})-\operatorname{div} \mathbb{T}(p, \mathbf{u})-\mathscr{G} \boldsymbol{\nabla}=\mathbf{0} \\
\operatorname{in} \bigcup_{i=1,2, v} \Omega_{i} \times(0, T), \\
\partial_{t} \phi+\operatorname{div}(\phi \mathbf{u})-\operatorname{div}(\omega \nabla \mathscr{G})=0 \\
\operatorname{in} \bigcup_{i=1,2, v} \Omega_{i} \times(0, T),
\end{gathered}
$$




$$
\begin{gathered}
\operatorname{div} \mathbf{u}=0 \\
\operatorname{in} \bigcup_{i=1,2, v} \Omega_{i} \times(0, T),
\end{gathered}
$$

where the stress tensor $\mathbb{T}(p, \mathbf{u})$, constructed with the pressure and velocity fields $p(\mathbf{r}, t), \mathbf{u}(\mathbf{r}, t)$ reads as follows:

$$
\begin{aligned}
& \mathbb{T}(p, \mathbf{u})=-p \rrbracket+2 \mu \mathbb{S}(\mathbf{u}), \\
& \text { where } \mathbb{S}(\mathbf{u})=\frac{1}{2}\left\{\nabla \mathbf{u}+(\nabla \mathbf{u})^{T}\right\} .
\end{aligned}
$$

It is understood that $\Omega_{v}=\Omega_{v}^{-} \cup \Omega_{v}^{+}\left(=\Omega_{v}^{ \pm}\right)$. In (11) and (12), the property constants $\rho, \mu>0$ characterise the mixed density and dynamic viscosity of the slug; both are defined in terms of the material properties of the two phases, and the smooth (phase-field) function $\phi_{12}(\mathbf{r}, t) \in[0,1]$ is $\beta=\beta_{v}+\left(\beta_{2}-\beta_{v}\right) \phi_{12}$ (with $\beta=\rho, \mu$ ). The function $\mathscr{G}(\phi)$ defines the so-called bulk chemical potential as

$$
\begin{aligned}
\mathscr{G}(\phi)=-\frac{\zeta}{\varepsilon^{2}}\left\{\operatorname{div}\left(\varepsilon^{2} \nabla \phi\right)-\left(\phi^{2}-1\right) \phi\right\}, \\
\text { where } \phi(\mathbf{r}, t) \in[-1,1] .
\end{aligned}
$$

In (13), $\varepsilon>0[\mathrm{~m}]$ is a capillary width that scales with the thickness of the boundaries $\Gamma_{1}, \Gamma_{2} ; \omega>0\left[\mathrm{~m}^{3} \cdot \mathrm{s} \cdot \mathrm{kg}^{-1}\right]$, the mobility term which determines the time scale of the CahnHilliard diffusion; and $\zeta>0[\mathrm{~N}]$, the mixing energy density. For more details see, for instance, the papers of Yue et al. [39] and Jacqmin [40] as well. We draw attention to the fact that $\phi_{12} \simeq(1+\phi) / 2$, and, therefore

$$
\phi_{12}=\min \left\{\max \left(\frac{1+\phi}{2}, 0\right), 1\right\} \text {, }
$$

where $\phi(\mathbf{r}, t) \in[-1,1]$.

In $(14), \min (\cdot)$ and $\max (\cdot)$ are operators which give the minimum and maximum of their respective arguments. Throughout the following discussion, the phase-field function $\phi(\mathbf{r}, t) \in[-1,1]$ is used instead of $\phi_{12}(\mathbf{r}, t) \in[0,1]$. It now remains to supplement the appropriate boundary conditions on $\Gamma_{1}, \Gamma_{2}, \Gamma_{s}$. At the inlet and outlet boundaries a zero pressure is imposed; that is, the system containing the domains $\Omega_{1}, \Omega_{2}$ is embedded in a still environment $\Omega_{v}$. Wetted wall boundary conditions are imposed along $\Gamma_{s}$; the contact angles are such that $\theta_{1} \equiv \theta_{2}(\equiv \pi / 3[\mathrm{rad}])$. The choice $\theta_{k} \equiv \pi / 3$ [rad] (with $k=1,2)$ is justified from the fact that the advancing and receding boundaries of the miscible bidroplet system $\Omega_{1} \cup \Omega_{2}$ are convex curves, following the works of Bico and Quéré $[1,5]$. More features based partly on the field $\phi(\mathbf{r}, t) \in[-1,1]$ are discussed in Section 3.

\section{Numerical Implementation}

This section furnishes the mathematical model so far discussed with numerical features to simulate the motion of the slug. Figure 3 shows a simplified computational box model

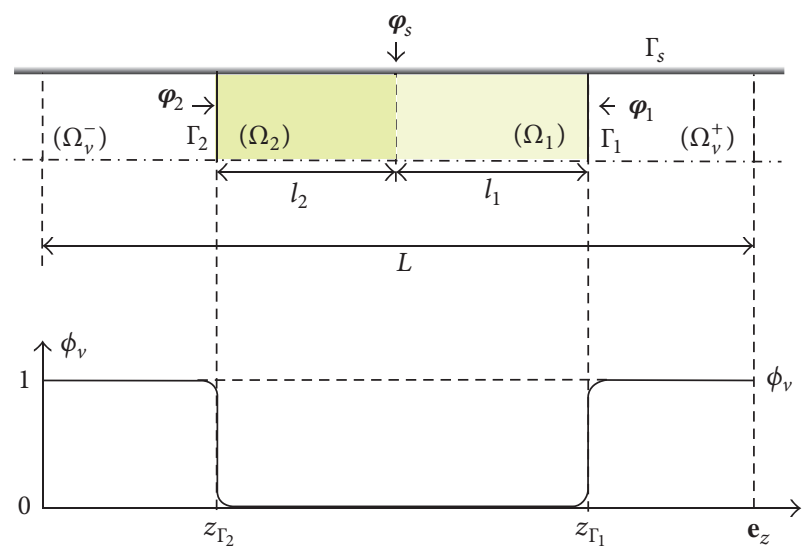

FIGURE 3: Sketch diagram of the computational box model used in this study paralleled with the corresponding zero level set function $\phi_{v}(z, t) \in[0,1]$ to delineate the regions of interest.

with the corresponding zero level set function $\phi_{v}(\mathbf{r}, t) \in[0,1]$ delineating the regions of interest.

Recall that we are concerned with the motion of a slug so in order to adapt the solution of $\mathscr{C}^{(l)}(\mathbf{r}, t)$ with its motion one should remap certain property variables attached to $\Omega_{1} \cup \Omega_{2}$ on a phase-field basis to track the evolution of this function while following the region $\Omega_{1} \cup \Omega_{2}$. In other words, this suggests to remap the scalar fields $\mathscr{C}^{(l)}(\mathbf{r}, t), \varphi_{k}$ as follows: $\mathscr{C}^{(l)} \mapsto \phi \mathscr{C}^{(l)}=\mathscr{C}_{\phi}^{(l)}, \varphi_{k} \mapsto \phi \varphi_{k}=\varphi_{\phi, k}$ (with $k=1,2, s$ ). Moreover, since $\phi \in[-1,1]$, which is equivalent to say that $\min \phi=-\max \phi=1$, the remapping $\phi \mapsto(1+\phi) / 2$ is first taken into account so as to obtain $\phi \in[0,1]$, as can be easily checked by substitution. Clearly, from these remappings one observes that the convectiondiffusion equation vanishes only outside the region $\Omega_{1} \cup \Omega_{2}$. Consequently, these now result in a model which spans over $\bigcup_{i=1,2, v} \Omega_{i}$, while retaining its characteristics only in $\Omega_{1} \cup \Omega_{2}$. Hence, invoking these mapping properties into (5) yields

$$
\begin{aligned}
\phi \partial_{t} \mathscr{C}^{(l)}+\operatorname{div} \Xi_{\phi}= & \mathscr{F}_{\phi} \text { in } \bigcup_{i=1,2, v} \Omega_{i} \times(0, T), \\
\mathscr{C}_{\phi}^{(l)}(\mathbf{r}, t)= & i-1 \\
& \text { in } \Omega_{i} \times\{t=0\} \quad(i=1,2), \\
-\mathbf{n}_{i} \cdot\left(\mathscr{C}_{\phi}^{(l)} \mathbf{u}-D_{21} \nabla \mathscr{C}_{\phi}^{(l)}\right)= & \varphi_{\phi, i}=-\mathbf{n}_{i} \cdot\left(\mathscr{C}_{\phi}^{(l)} \mathbf{u}\right) \\
& \text { on } \Gamma_{i} \times(0, T) \quad(i=1,2, s),
\end{aligned}
$$

where the fields $\Xi_{\phi}(\mathbf{r}, t), \mathscr{F}_{\phi}(\mathbf{r}, t)$ are for shortness defined by

$$
\begin{aligned}
& \Xi_{\phi}(\mathbf{r}, t)=\left(\mathbf{u}-D_{21} \nabla\right) \mathscr{C}_{\phi}^{(l)} \text { in } \bigcup_{i=1,2, v} \Omega_{i} \times(0, T) ; \\
& \mathscr{F}_{\phi}(\mathbf{r}, t)=-\left(\partial_{t} \phi\right) \mathscr{C}^{(l)} \text { in } \bigcup_{i=1,2, v} \Omega_{i} \times(0, T) .
\end{aligned}
$$

Following (15), the problem then becomes one of finding the solution of a General Form PDE. Alternatively, one can 
formulate the convection-diffusion equation describing the scalar field $\mathscr{C}^{(l)}(\mathbf{r}, t)$ as follows:

$$
\begin{aligned}
\partial_{t} \mathscr{C}^{(l)}+\operatorname{div}\left(\phi \mathbf{u} \mathscr{C}^{(l)}-D_{21} \phi \nabla \mathscr{C}^{(l)}\right) & =\mathscr{R}_{\phi}(\mathbf{r}, t) \\
& \text { in } \bigcup_{i=1,2, v} \Omega_{i} \times(0, T),
\end{aligned}
$$

where the reaction term $\mathscr{R}_{\phi}(\mathbf{r}, t)$ appearing in the above transport reads as

$$
\begin{aligned}
\mathscr{R}_{\phi}(\mathbf{r}, t)=(1-\phi) \partial_{t} \mathscr{C}^{(l)}-\left(\partial_{t} \phi\right) \mathscr{C}^{(l)} & \\
& \text { in } \bigcup_{i=1,2, v} \Omega_{i} \times(0, T) .
\end{aligned}
$$

This is the problem of finding the solution of a Transport PDE. In COMSOL, the latter is called Transport of Dilute Species Interface. It may be observed in passing the fact that whenever $\mathbf{r}=(r, z)^{T}$ is a point of $\Omega_{1} \cup \Omega_{2},(1-\phi)=0$ implies that $\partial_{t} \phi=0$ and $\mathscr{C}_{\phi}^{(l)}=\mathscr{C}^{(l)}$, thence the original convectiondiffusion equation. Contrarily, when $\mathbf{r}=(r, z)^{T}$ is not a point of $\Omega_{1} \cup \Omega_{2},(1-\phi)=1$ implies that $\partial_{t} \phi=0$ and $\mathscr{C}_{\phi}^{(l)}=0$, thence the restriction of the property $\mathscr{C}^{(l)}(\mathbf{r}, t)$ within the domain $\Omega_{1} \cup \Omega_{2}$. These latter two statements confirm very clearly the validity of the above underlying mapping procedures.

Now, some techniques which have rendered efficient aid towards implementing this model with COMSOL are in order. Henceforth, we presume the following COMSOL interfaces are activated: the phase-field and General Form $P D E$ interfaces, respectively: the former for the NS- and $\mathrm{CH}$-models and the latter for the CD-model. Decidedly, it is observed that the conditions imposed on $\mathscr{C}^{(l)}(\mathbf{r}, t)$ imply that

$$
\left.\left.\mathscr{C}_{\phi}^{(l)}\right\rfloor_{\Gamma_{2}} \geq \mathscr{C}_{\phi}^{(l)}\right\rfloor_{\Gamma_{1}} \geq 0 \quad \forall t \in(0, T)
$$

thence, it follows from (19), using the $\min (\cdot)$ and $\max (\cdot)$ operators, that

$$
\left.\mathscr{C}_{\phi}^{(l)}\right\rfloor_{\Gamma_{k}} \equiv \begin{cases}\max _{\mathscr{C}_{\phi}^{(l)}}(\mathbf{r}, t) & \text { for } t \in(0, T) \text { if } k=2 \\ \min \mathscr{C}_{\phi}^{(l)}(\mathbf{r}, t) & \text { for } t \in(0, T) \text { if } k=1 .\end{cases}
$$

With these features alongside, we first utilise the COMSOL $\min (\cdot)$ and $\max (\cdot)$ operators to construct the interfacial forces $\gamma_{i}\left(\mathscr{C}_{\Gamma_{i}}^{(l)}\right)$ at their respective boundaries $\Gamma_{1}, \Gamma_{2}$. Then, these and other fluid properties are entered into the fluid properties $i$ (with $i=1$,2) features. Finally, $\Gamma_{1}, \Gamma_{2}$ are selected as initial interfaces. In regard to the General Form PDE interface, (15), its coefficients and flux components, namely, $\partial_{\lambda} \Xi(\mathbf{r}, t)$ (with $\lambda=r, z$ ), are entered into the appropriate default nodes, the concentration in $\Omega_{2}$ is initialized to unity, and a zero flux boundary condition is assumed from the surroundings. This being so, several simulations were then run with an array of data extracted from the papers of Bico and Quéré $[1,5]$. Extracted data from those authors are tabulated in Table 1. These simulations are discussed through several cases in the following sections.
TABLE 1: The set of property constants used to run the numerical experiments: data used for case studies 1 to 4 .

\begin{tabular}{lcccc}
\hline \multicolumn{5}{c}{ Data used for case studies } \\
\hline$R$ & $\left(l_{1}, l_{2}\right)$ & $\left(\rho_{1}, \rho_{2}\right)$ & $\left(\mu_{1}, \mu_{2}\right)$ & $\left(\gamma_{1,0}, \gamma_{2,0}\right)$ \\
{$[\mathrm{mm}]$} & {$[\mathrm{mm}]$} & {$\left[\mathrm{g} \cdot \mathrm{cm}^{-3}\right]$} & {$\left[\mathrm{kg} \cdot \mathrm{m}^{-1} \cdot \mathrm{s}^{-1}\right]$} & {$\left[\mathrm{mN} \cdot \mathrm{m}^{-1}\right]$} \\
$4 \times 10^{-1}$ & $(15,15)$ & $(1.1132,0.95)$ & $(1.6,1.7) \times 10^{-2}$ & $(47.7,20.3)$ \\
\hline
\end{tabular}

\section{Results and Discussion}

In this section we analyse the results of our simulations based on the data given in Table 1. The data appearing in Table 1 are those extracted from the papers of Bico and Quéré $[1,5]$. This made the need of a condensed notation imperative. Define in compact form those property constants, namely, $R, l_{s}, D_{21}, \mu>0$, upon which the numerical experiments were carried out as $\pi \stackrel{\text { def }}{=}\left(R, l_{s}, D_{21}, \mu\right)^{T}$, and henceforward view the surface tension difference between both menisci as a function of the set of parameters $\pi$ and of time $t>0$, respectively; that is,

$$
\gamma(t, \pi)=\gamma_{1}(t, \pi)-\gamma_{2}(t, \pi)
$$

for given $\pi, t \in(0, T)$.

Let the position and speed/velocity, that is, $X_{s}(t, \pi)$ and $U_{s}(t, \pi)$, respectively, of the slug be functions of the forms

$$
\Xi_{s}=\Xi_{s}(t, \gamma) \text { for } \Xi_{s}=X_{s}, U_{s} \text {; given } \pi, t \in(0, T),
$$

so that the corresponding differentials with respect to time write as

$$
\begin{array}{r}
\frac{d \Xi_{s}}{d t}(\mathbf{r}, t)=\left(\partial_{t}+\partial_{t} \gamma \frac{d}{d \gamma}\right) \Xi_{s}(\mathbf{r}, t)=\mathscr{L}_{\gamma}\left[\Xi_{s}\right](\mathbf{r}, t) \\
\text { for } \Xi_{s}=X_{s}, U_{s} .
\end{array}
$$

From now on, we can then characterise $\partial_{t} \Xi_{s}$ as the instantaneous rate of change but $d \Xi_{s} / d \gamma$, the net change in the property $\Xi_{s}(t, \gamma)$ with respect to interfacial variation and $\partial_{t} \gamma(t, \pi)$, the net rate of change in interfacial forces with respect to time. In particular, by $\mathscr{L}_{\gamma}\left[\Xi_{s}\right](\mathbf{r}, t)$ is meant the time derivative of $\Xi_{s}(t, \gamma)$ following the dynamics of $\gamma(t, \pi)$, where $\Xi_{s}=$ $X_{s}(t, \pi), U_{s}(t, \pi)$. We will come back to those terms in later sections. In other words, these bring forward the key factors that do have significant effects on the evolution of the scalar fields $X_{s}(t, \pi), U_{s}(t, \pi)$. Suppose the following baseline properties are granted: $\pi^{(0)}=\left(R^{(0)}, l_{s}^{(0)}, D_{21}^{(0)}, \mu^{(0)}\right)^{T}$, and important questions related to those properties might then be addressed. For instance, it would be fruitful to extend theoretical understanding based on the following inequalities:

$$
\begin{aligned}
& \beta \leq \beta^{(0)}, \\
& \beta>\beta^{(0)}
\end{aligned}
$$




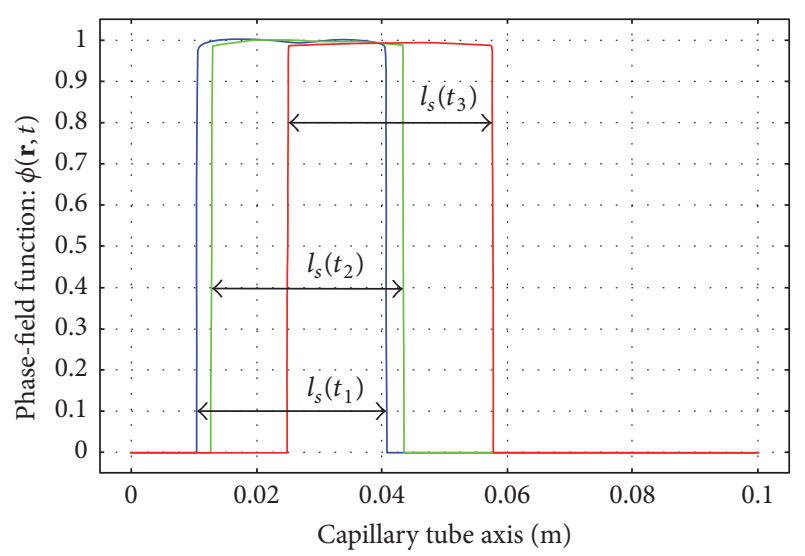

Figure 4: Plots of the phase-field function $\phi(\mathbf{r}, t)$ along the axis of the capillary tube at the sequence of times $\left(t_{k}\right)_{k=1,2,3}=\left(5^{k-1}\right)_{k=1,2,3}$ [s].

Henceforth, we primarily aim at answering two sets of questions. In the first place, we aim to answer the following questions.

Question 2 (given $\pi^{(0)}$ ). What are the kinematics of the slug in steady and unsteady regimes?

Question 3 (given $\pi^{(0)}$ ). How does the slug evolve when $\pi^{(0)}$ is perturbed to $\pi^{(0)}+\delta \pi$ ?

And, in the second place, we aim to answer the following question.

Question 4 (given $\pi^{(0)}$ and $\left(t_{\infty}, X_{s, \infty}\right)$, resp.). Are the positions of the slug predictable for all $t \in\left(t_{0}, t_{\infty}\right)$ ?

In the above last question, the scalar field $X_{s, \infty}>0$ denotes the position of the miscible fluids system after selfpropulsion and $t_{\infty} \leq T$ the corresponding time. Thus, by the pairs of scalars $\left(t_{0}, X_{s, 0}\right),\left(t_{\infty}, X_{s, \infty}\right)$ are meant the ending points of its path in the $\left(t, X_{s}\right)$-plane. In the following sections we shed lights on the above three questions by advancing a series of graphical results and mechanical explanations.

\section{The Phase-Field Function}

The proposed methodological approach rests on the phasefield technique. Knowing the subsequent positions of the phase-field function $\phi(\mathbf{r}, t)$ when time evolves, one can take advantage if it calculates the subsequent position of the slug. Thus, the discussion commences by computing the scalar field $\phi(\mathbf{r}, t)$. To do so, we use the set of property constants tabulated in Table 1.

The time evolution of the phase function $\phi(\mathbf{r}, t)$ is illustrated at the sequence of times $\left(t_{k}\right)_{k=1,2,3}=\left(5^{k-1}\right)_{k=1,2,3}[\mathrm{~s}]$ in Figure 4. We draw attention that $\phi(\mathbf{r}, t)$ is graphed by the remapping $\phi \mapsto(1+\phi) / 2$ in order to have $\phi \in[0,1]$ instead of $\phi \in[-1,1]$.

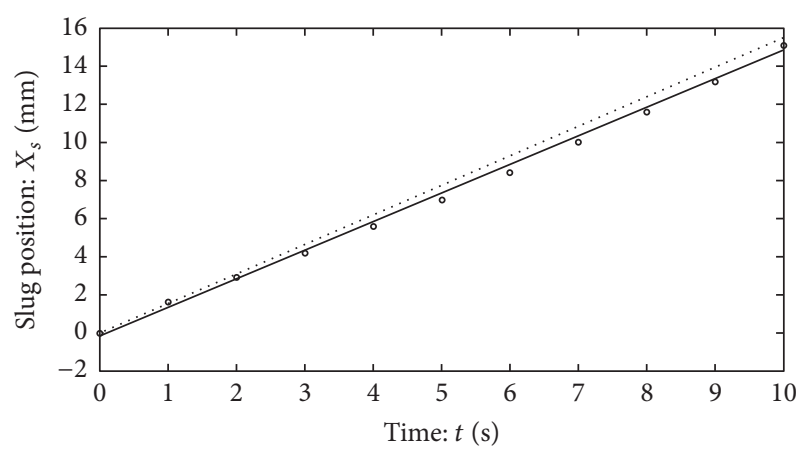

FIgURE 5: Position of the slug as function of time, $X_{s}(t) \approx 1.5 t-$ $0.15[\mathrm{~mm}]$. The radius of the capillary tube is $0.4[\mathrm{~mm}]$, and the successive lengths are $l_{1}=16[\mathrm{~mm}]$ and $l_{2}=11[\mathrm{~mm}]$. Note that the experimental result gives $U_{s} \approx 1.55\left[\mathrm{~mm} \cdot \mathrm{s}^{-1}\right]$, and ours reads as $U_{s} \equiv \partial_{t} X_{s} \approx 1.50\left[\mathrm{~mm} \cdot \mathrm{s}^{-1}\right]$. The numerical result (solid line) is compared with that of Bico and Quéré [5] (dotted line).

\section{Case 1: Motion of the Slug versus Time}

The following two cases concern the rate of penetration of the slug into the capillary tube in steady and unsteady regimes. The steady case/regime refers to the case for which the position-time graph of the slug is linear and the unsteady case/regime to other cases.

6.1. Case 1.1: Steady Regime. Generally, on a Poiseuille flow hypothesis together with the assertion that the slug is sufficiently long, $l_{s} \gg 1$ say, its path results in an integral expression of the form

$$
X_{s}(t, \gamma)=\int \frac{\left(\gamma_{1}-\gamma_{2}\right) R}{4\left(\mu_{1} l_{1}+\mu_{2} l_{2}\right)} d t+\text { Const. }
$$

for given $\pi, t \in(0, T)$.

To settle (25), we have taken into account the following statements [1]: the Poiseuille flow induced within the region $\Omega_{1} \cup \Omega_{2}$ is of the form $u_{r}=\delta p\left(R^{2}-r^{2}\right) / 4 \mu_{s} l_{s}$, where the Laplacian pressure exerted at $\Gamma_{1}$ relative to that exerted at $\Gamma_{2}$ reads as $\delta p=2\left(\gamma_{1}-\gamma_{2}\right) / R$. The velocity field $u_{r}$ is averaged in $\Omega_{1} \cup \Omega_{2}$, and the resulting expression equated to $d X_{s} / d t$, yielding $d X_{s} / d t=R\left(\gamma_{1}-\gamma_{2}\right) / 4\left(\mu_{1} l_{1}+\mu_{2} l_{2}\right)$, since $\mu l_{s}=$ $\mu_{1} l_{1}+\mu_{2} l_{2}$, thence the antiderivative of (25).

Consequently, it follows that there exists a functional $\chi(t, \pi)$ such that $X_{s}(t, \gamma)=\int \chi(t, \pi) d t+$ Const. for given $\pi=$ $\left(R, l_{s}, D_{21}, \mu\right)^{T}, t>0$. Numerical simulation was carried out for the steady case in the hope of establishing the character of $\chi(t, \pi)$. The early-time results confirm that $\chi=\chi(\pi)$, and the position of the slug obeys the following linear law at earlytime:

$$
\begin{aligned}
X_{s}(t, \gamma) \approx \chi(\pi) \cdot\left(t-t_{0}\right), \\
\chi(\pi) \approx \text { Const. for } t \in\left(t_{0}, t_{10}\right) \subset(0, T),
\end{aligned}
$$

where $t_{10}=10$ [s], as can be easily seen in Figure 5. To graph Figure 5, the COMSOL Cut Line data set is used to create a 
line parallel to the axis of the capillary tube, having its ending points at $(r, z)=(R / 2,0),(R / 2, L)$, where $L \gg l_{s}$ denotes the length of the capillary tube along the $z$-axis. This allows to track the subsequent positions occupied by the advancing and receding boundaries, that is, $\Gamma_{1}, \Gamma_{2}$, since it will cut at every instant of time $t \in(0, T)$ these boundaries at precisely two and only two (boundary) points $\mathbf{r}=\left(R / 2, z_{\Gamma_{k}}(t)\right)^{T} \in \Gamma_{k}$ (with $k=1,2$ ). Setting $X_{s}=z_{\Gamma_{1}}(t)$, we then construct a timeposition sequence $\left(t_{k}, X_{s}\left(t_{k}, \pi\right)\right)_{k \in[0,10]}=\left(k, X_{s, k}\right)_{k \in[0,10]}$. Representing these data points by circular markers $(\circ)$, the corresponding approximation by solid line, and that of Bico and Quéré [5] by dotted line, one obtains Figure 5. Henceforth, whenever the study is carried out with respect to time, it is to be understood that the plotting referred to in the $\left(t, X_{s}\right)$-plane is based on that approach, unless the study is carried out parametrically, in which case we shall then draw attention upon.

In the sequel, we will show through numerical experiments that $\chi(\boldsymbol{\pi}) \approx R\left(\gamma_{1}-\gamma_{2}\right) / 4\left(\mu_{1} l_{1}+\mu_{2} l_{2}\right)$. Thus, by (26) it would appear that after some time $t>t_{0}$, the slug will have travelled at a constant velocity $U_{s} \equiv \chi(\pi)$ a distance $X_{s}(t, \gamma) \approx \chi(\pi) \cdot\left(t-t_{0}\right)$. Clearly, (26) appears to show that $X_{s} \rightarrow \infty$ whenever $t \rightarrow \infty$, a situation which is reasonable mathematically but unreasonable physically; this is simply because the mathematical description using the linear approximation $X_{s}(t, \gamma) \approx \chi(\pi) \cdot\left(t-t_{0}\right)$ will at some time $t>0$ (unsteady case) fail to give physical agreement. In that case a nonlinear law (as will be shown in the sequel to exist) should be sought.

The above statements suggest that there exists a scalar $X_{s}^{*} \in(0, \infty)$, characterising the distance at which the slug stops, such that $\lim _{t \rightarrow \infty} X_{s}(t, \gamma) \rightarrow X_{s}^{*}$, since we know from the nature of the problem that the difference in interfacial forces, which was initially very high, that is, $\gamma_{1,0}-\gamma_{2,0}\left(\equiv \gamma^{(0)}\right)$, owing to the initial conditions $\mathscr{C}^{(l)}(\mathbf{r}, t=0)=i-1$ in $\Omega_{i}$ (with $i=1,2$ ), will have decreased to a vanishingly small value when the equilibrium of $\mathscr{C}^{(l)}(\mathbf{r}, t)$ has been established. Thus, this demonstrates the existence of a temporal ( $\boldsymbol{\pi}$-dependant) property $t_{\infty} \in(0, T)$ such that mechanical equilibrium is attained via interfacial effects; namely,

$$
\gamma_{1}(t, \pi) \approx \gamma_{2}(t, \pi)
$$

whenever $t \gtrsim t_{\infty} \in(0, T) ; \pi$ given.

Clearly, there is no loss of generality in assigning, for instance, this particular case to an initially prescribed set, say $\beta \equiv \beta^{(0)}$, where $\beta=R, l_{s}, D_{21}, \mu$. Based on the said facts, we ran the numerical simulation with a large final time $T=100[\mathrm{~s}]$ to demonstrate that (27) is satisfied.

Surprisingly, we observed with a slug bearing the characteristics $l_{s}=30[\mathrm{~mm}], \mu_{k} \approx 17[\mathrm{mPa} \cdot \mathrm{s}]$ (with $k=1,2$ ), and $D_{21} \approx 10^{-9}\left[\mathrm{~m}^{2} \cdot \mathrm{s}\right]$ - the diffusion constant for alcohol fluid 1 in water fluid $2[41,42]$ - that

$$
\begin{aligned}
& \gamma\left(t_{\infty}, \pi\right) \leq 5 \% \\
& \quad \text { of } \min \left(\gamma_{1,0}, \gamma_{2,0}\right) \text { for given } \pi, t_{\infty} \gtrsim 100[\mathrm{~s}] .
\end{aligned}
$$

The graphical evolution of the surface tension function $\gamma(t, \pi)$ with respect to time $t \in\left[t_{0}, t_{\infty}\right)$ is shown in Figure 6. When

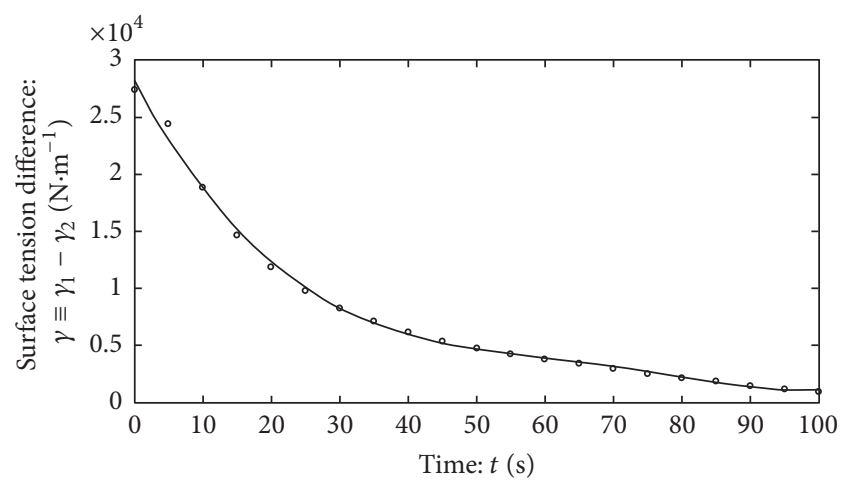

FIGURE 6: Surface tension difference versus time: $\gamma(t, \pi)\left[\mathrm{N} \cdot \mathrm{m}^{-1}\right]$. The radius of the capillary tube is $0.4[\mathrm{~mm}]$; the length of the slug is such that $l_{s}=l_{1}+l_{2} \approx 15 l_{s}+15 l_{s}[\mathrm{~mm}]$ (with both the scalar fields $l_{s}, \pi$ kept fixed $)$. Observe that $\gamma\left(t_{\infty}, \pi\right) \lesssim 5 \%$ of $\min \left(\gamma_{1,0}, \gamma_{2,0}\right)$ for $t_{\infty} \gtrsim 100[\mathrm{~s}]$.

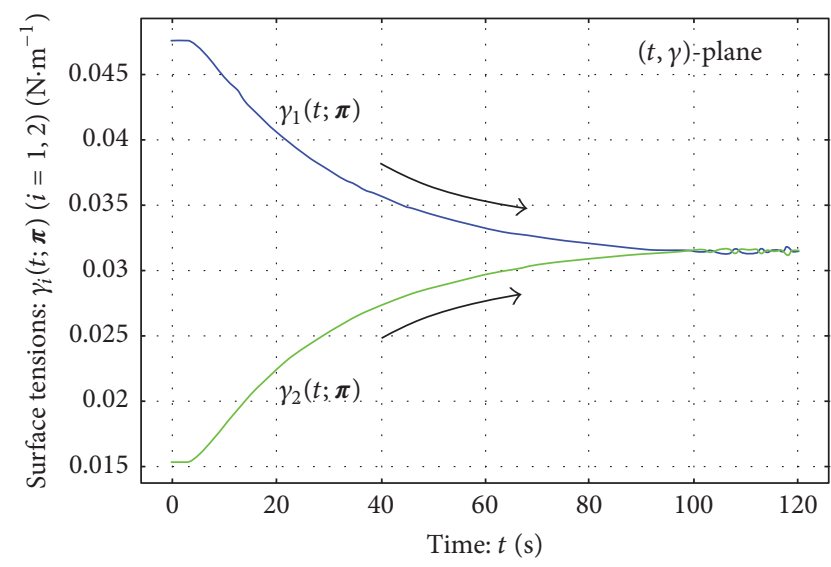

FIGURE 7: The time evolution of the surface tensions $\gamma_{i}(t ; \pi)(i=1$, 2).

the surface tensions $\gamma_{i}(t ; \pi)(i=1,2)$ are plotted on the same $(t, \gamma)$-plane, one obtains Figure 7 , where curved arrows appearing in that plane denote the direction of convergence of these functions with respect to time.

A very interesting feature is observed from Figure 7. We have $\gamma_{1}(t ; \pi) \approx \gamma_{2}(t ; \pi) \approx 3.2 \times 10^{-2}\left[\mathrm{~N} \cdot \mathrm{m}^{-1}\right]$ at time $t \approx$ $100[\mathrm{~s}]$. Substituting the values tabulated in Table 1 into the barycentric-model equation (10) for the pairs of scalar fields $\left(l_{1}, l_{2}\right),\left(\gamma_{1,0}, \gamma_{2,0}\right)$, one arrives at $\gamma_{i, \infty} \approx 3.3 \times 10^{-2}\left[\mathrm{~N} \cdot \mathrm{m}^{-1}\right]$ (with $i=1,2$ ), thereby validating the form of (10).

Out of the graphical results displayed in Figures 6 and 7 , it would appear that (26) does not hold in both the earlyand late-time regimes, more precisely, in the steady (or earlytime) regime as well as in the unsteady (or late-time) regime. The next section discusses the dynamics of the slug in the unsteady regime.

6.2. Case 1.2: Unsteady Regime. When the period over which the numerical experiments were extended to $\left[t_{0}, \leqslant 150\right.$ ) [s], the subsequent positions of the slug graphed exponentially, as can be easily shown in Figure 8. Hence, the path of the slug 


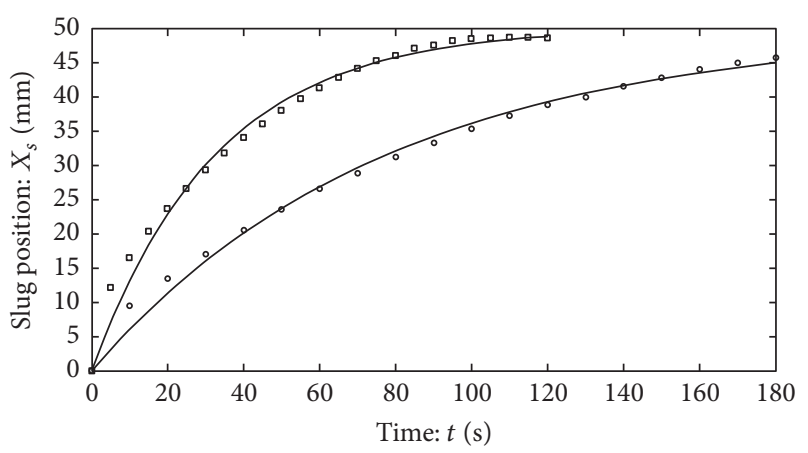

FIGURE 8: Slug position versus time, described by (31) and compared with numerical results (empty squares $(\square)$ for $l_{s}=30[\mathrm{~mm}]$ and empty circles (०) for $\left.l_{s}=60[\mathrm{~mm}]\right)$.

could fairly well be described by an exponential-type equation of the form

$$
\begin{aligned}
& X_{s}(t, \pi) \approx \eta_{1}\left\{1-\exp \left(-\eta_{2}\left(t-t_{0}\right)\right)\right\}, \\
& \text { for given } \pi, t \in(0, T) .
\end{aligned}
$$

In (29), the fields $\eta_{1}, \eta_{2}>0$ are $\pi$-dependent scalars; that is, $\eta_{1}=\eta_{1}(\boldsymbol{\pi})$ and $\eta_{2}=\eta_{2}(\boldsymbol{\pi})$. Furthermore, since the slug moves steadily only for a short period of time $\left[0, t_{10}\right)$ (roughly, $t_{10} \approx$ $10[\mathrm{~s}]$ ), consequently, there does necessarily exist $X_{s, \infty}, U_{s}>0$ such that, for given $\pi=\left(R, l_{s}, D_{21}, \mu\right)^{T}$,

$$
\begin{aligned}
& \lim _{t \rightarrow t_{\infty}} X_{s}(t, \pi) \longrightarrow X_{s, \infty}, \\
& \lim _{t \rightarrow t_{10}} \partial_{t} X_{s}(t, \pi) \longrightarrow U_{s}, \\
& \lim _{t \rightarrow t_{\infty}} \partial_{t} X_{s}(t, \pi) \longrightarrow 0,
\end{aligned}
$$

where $0 \leq t_{0}<t_{10}<t_{\infty} \leq T$. Here, it is to be understood that $X_{s, \infty}>0$ is the place at which the slug stops whereas $U_{s}>0$ simply characterises its steady regime. Also, $t_{0}, t_{10}, t_{\infty} \geq 0$ stand, respectively, for the initial time $t_{0}=0[\mathrm{~s}]$, the time $t_{10}=10[\mathrm{~s}]$, and the time $t_{\infty} \leq T[\mathrm{~s}]$ at which the slug stops. But $T>0$ designates the period over which the numerical experiments are carried out. With these characteristics at hand, the path of the slug then writes as

$$
\begin{aligned}
X_{s}(t, \pi) & \approx X_{s, \infty}\left\{1-\exp \left(-\frac{U_{s}}{X_{s, \infty}}\left(t-t_{0}\right)\right)\right\} \\
& \equiv U_{s}\left(t-t_{0}\right)-\sum_{k=2}^{\infty} \frac{U_{s}^{k}}{X_{s, \infty}^{k-1} k !}\left(t_{0}-t\right)^{k} \\
& \equiv X_{s, 0}(t, \pi)+x_{s}(t, \pi)
\end{aligned}
$$

for given $\pi, t \in(0, T)$.

From this, one concludes that the position $X_{s}(t, \pi)$ of the slug is merely a superposition arising from two contributions: the steady and unsteady regimes; the former is characterised by $X_{s, 0}(t, \pi)$ and the latter by $x_{s}(t, \pi)$, respectively. Following Figure 5 , it is obvious that the equality $X_{s, 0}=X_{s}(t, \pi)$ holds
TABLE 2: Numerical values for $\left(\eta_{1}, \eta_{2}\right)$, data for (29).

\begin{tabular}{lcc}
\hline \multicolumn{3}{c}{ Results of case study 1.2} \\
\hline$\left(\eta_{1}, \eta_{2}\right)$ & $l_{s}=30[\mathrm{~mm}]$ & $l_{s}=60[\mathrm{~mm}]$ \\
$\eta_{1}[\mathrm{~mm}]$ & 50.0542 & 49.8052 \\
$\eta_{2}\left[\mathrm{~s}^{-1}\right]$ & 0.0306 & 0.0129 \\
$U_{s} \approx \eta_{1} \cdot \eta_{2}\left[\mathrm{~mm} \cdot \mathrm{s}^{-1}\right]$ & 1.5312 & 0.6425 \\
\hline
\end{tabular}

only for $t \in\left[t_{0}, 10\right)[\mathrm{s}]$. Thus, for early times, the position of the slug can be approximated as

$$
\begin{aligned}
& X_{s}(t, \pi) \approx U_{s}\left(t-t_{0}\right) \\
& \quad \text { for given } \pi, t \in\left(0, t_{10}\right) \subset(0, T),
\end{aligned}
$$

which is precisely the same expression in the steady case, $U_{s}>$ 0 being put for $\eta_{1} \cdot \eta_{2}>0$. It now remains to demonstrate that $U_{s}\left(\approx \eta_{1} \cdot \eta_{2}\right)$ coincides with the result of Bico and Quéré [5].

For two typical slugs of lengths $l_{s}=30,60[\mathrm{~mm}]$, respectively, we combined (29) with numerical data in the leastsquares sense. We found $\left(\eta_{1}, \eta_{2}\right) \approx(50.0542,0.0306)$ for $l_{s}=$ $30[\mathrm{~mm}]$ and $\left(\eta_{1}, \eta_{2}\right) \approx(49.8052,0.0129)$ for $l_{s}=60[\mathrm{~mm}]$. Thence, with the former, we deduce $U_{s} \approx 1.5312\left[\mathrm{~mm} \cdot \mathrm{s}^{-1}\right]$ and with the latter $U_{s} \approx 0.6425\left[\mathrm{~mm} \cdot \mathrm{s}^{-1}\right]$. All these quantities are arranged carefully in Table 2 . Figure 8 is intended to exhibit these features graphically using (31).

No doubt, it is no exaggeration to say that our model, verified numerically in those two cases, namely, for $l_{s}=$ 30, $60[\mathrm{~mm}]$, as depicted in Figure 8, may be extended to analyse the movement of small volumes of viscous liquids in capillary tubes of square, rectangular cross sections and so forth. Hence, given the set $\left(X_{s, \infty}, U_{s}\right)$, that pair of scalar fields designating the point at which the slug stops for an initially given speed, one can predict a priori the position as well as the rate of penetration of the slug into the capillary tube. Further, if the focus is on rapid motion, one should evidently consider a small slug, instead of a big one. Contrarily, if the primary goal is to achieve large penetrating length, one might endeavour to take a large slug; the trueness of these two statements is reflected in Figure 8. The following sections enable further physical relationships to be grasped.

\section{Case 2: Motion of the Slug versus Geometrical and Physical Properties}

The position of the slug is such that $X_{s}=X_{s}(t, \pi)$. So far, we studied this position function with respect to time, keeping the elements of the parameter vector $\pi=\left(R, l_{s}, D_{21}, \mu\right)^{T}$ fixed at those values which are tabulated in Table 1 . Here it is that this function is studied parametrically. We agree that parametric studies are best undertaken when the property in question is time-invariant. We have, on the one hand, $\lim _{t \rightarrow t_{\infty}} X_{s}(t, \pi) \rightarrow X_{s, \infty}=$ Const. and $\lim _{t \rightarrow t_{10}} \partial_{t} X_{s}(t, \pi) \rightarrow$ $U_{s}=$ Const.; see (30). And, on the other hand, the slug moves according to Poiseuille flow only in the early-time regime. As a consequence, it seems, therefore, legitimate to carry out the parametric studies in the early-time regime, which are what we proposed to consider hereinafter. 


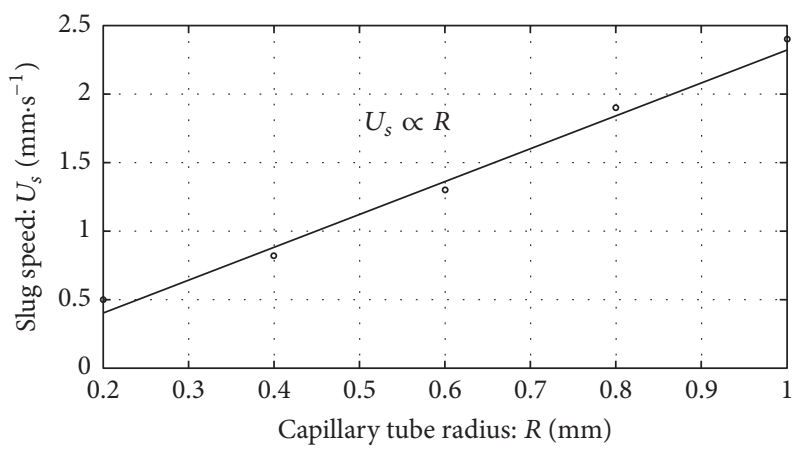

Figure 9: Plots of the sequence of points $\left(R^{(k)}, U_{s}^{(k)}\right)_{k=1}^{5}$. The linear expression describing the solid line is $U_{s} \approx 2.4 R-0.08$. The properties of the slug are $l_{1}=16[\mathrm{~mm}], l_{2}=16.5[\mathrm{~mm}]$, and $\mu_{k}=17$ [mPa.s] (with $k=1,2$ ). Other properties used are those given in Table 1.

Let $\mu_{s}>0$ designate the dynamic viscosity of the slug (mixture of the two miscible liquid droplets). On a barycentric-based basis we may then consider $\mu \cdot l_{s}=\mu_{1} l_{1}+\mu_{2} l_{2}$, in which case the velocity $U_{s}(t, \pi)$ of the slug, following (29), writes as

$$
U_{s}(t, \pi) \approx \frac{R\left(\gamma_{1}-\gamma_{2}\right)}{4\left(\mu_{1} l_{1}+\mu_{2} l_{2}\right)} \approx \frac{R\left(\gamma_{1}-\gamma_{2}\right)}{4 \mu l_{s}}
$$

for given $\pi, t \in(0, T)$.

We understand that (33) is the derivative of (25) with respect to $t \in(0, T)$; that is, $\partial_{t} X_{s}=U_{s}(t, \pi)$. If (33) holds true, so are the following proportionalities: $U_{s}(t, \pi) \propto R, \mu^{-1}$, and $l_{s}^{-1}>0$. These are what we are going to show through a series of numerical experiments in the following sections.

7.1. Case 2.1: Motion of the Slug versus the Radius of the Capillary Tube. In this section the motion of the slug is studied in a series of capillary tubes having the following radii: $R^{(k)}=(2 k) \times 10^{-1}[\mathrm{~mm}]$ (with $\left.k \in[1,5]\right)$. The properties of the slug are unaltered. We aim at establishing an algebraic expression relating the velocity $U_{s}$ of the slug to the radius $R$ of the capillary tube. For every $k \in[1,5]$, a numerical experiment is carried out and the velocity $U_{s}^{(k)}>0$ of the slug is calculated. When, for each $k \in[1,5]$, the ratio $U_{s}^{(k)} / R^{(k)}$ is analysed, it was found that

$$
\frac{U_{s}^{(k)}}{R^{(k)}} \approx 2.4 \times 10^{-2} \pm 5 \%\left[\mathrm{~s}^{-1}\right] \quad \forall k \in[1,5],
$$

from which we conclude that $U_{s} \propto R$. The corresponding pairs of values $\left(R^{(k)}, U_{s}^{(k)}\right)_{k=1}^{5}$ are plotted on a $\left(R, U_{s}\right)$-plane; see Figure 9. Consequently, the dependence of the functional $\chi(\pi)$ finally restricted to $\left(l_{s}, D_{21}, \mu\right) \equiv$ Consts evolves linearly with respect to the radius $R$ of the capillary tube according to the following linear relationship:

$$
\chi(\pi) \approx\left|K_{R}\right| R+K_{R, 0}, \quad \text { where } K_{R}, K_{R, 0}=\text { Consts. }
$$

It is understood that the scalar fields $K_{R}, K_{R, 0}$ are partially $\pi$ dependant constants (see the character of (33) to understand
TABLE 3: Comparison of $X_{s}(t, \pi)=\chi(\pi) \cdot\left(t-t_{0}\right)$ in the early-time regime.

\begin{tabular}{lccc}
\hline \multicolumn{4}{c}{ Results of case study 2.2 } \\
\hline$l_{s}$ & $X_{s}(t)$ & $U_{s} \equiv \chi(\pi)$ & Bico and Quéré $[5]$ \\
{$[\mathrm{mm}]$} & {$[\mathrm{mm}]$} & {$\left[\mathrm{mm} \cdot \mathrm{s}^{-1}\right]$} & {$\left[\mathrm{mm} \cdot \mathrm{s}^{-1}\right]$} \\
30 & $1.7 t-0.29$ & 1.7 & 1.6 \\
40 & $1.1 t+0.76$ & 1.1 & 1.0 \\
50 & $0.89 t+0.76$ & 0.89 & 0.8 \\
60 & $0.77 t+0.82$ & 0.77 & 0.7 \\
70 & $0.69 t-0.82$ & 0.69 & 0.65 \\
80 & $0.57 t+0.88$ & 0.57 & 0.6 \\
90 & $0.53 t-0.79$ & 0.53 & 0.5 \\
100 & $0.48 t+0.8$ & 0.48 & 0.4 \\
\hline
\end{tabular}

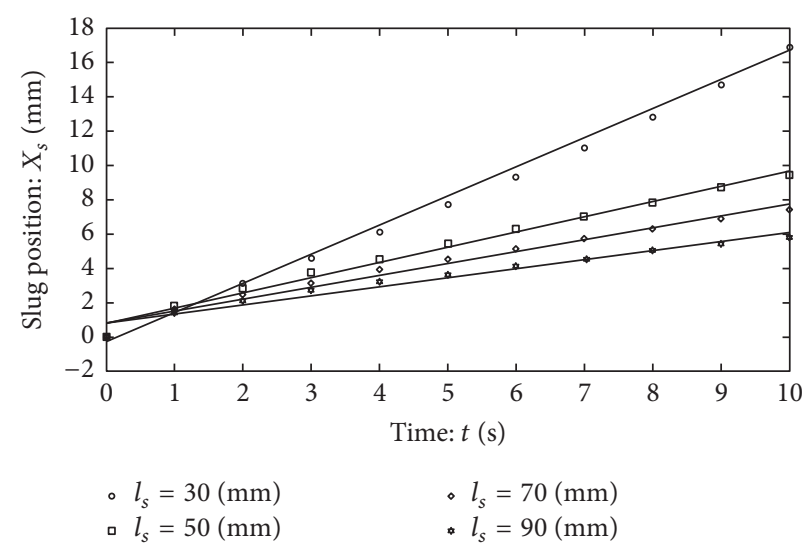

FIGURE 10: Position of the slug restricted to $l_{s}=30,50,70,90[\mathrm{~mm}]$ (with $l_{k}=l_{s} / 2, k=1,2$ ) as function of time (and of the property $\pi$ ): $X_{s}(t, \pi)$. The radius of the capillary tube is $0.4[\mathrm{~mm}]$.

this point). In other words, an increase in $R$ by $\delta R>0$ results in a linear increase in $U_{s}>0$, conversely, which manifests the nature of the size of the capillary tube. The next section examines the influence of the geometrical property $l_{s}>0$ on the kinematics of the slug.

7.2. Case 2.2: Motion of the Slug versus Its Length. We herein explore the effect of the geometrical property $l_{s}>0$ (size of the slug) on the slug motion. We ran for the sequence $l_{s}=30,40, \ldots, 100[\mathrm{~mm}]$ (with $l_{k}=l_{s} / 2, k=1,2$ ) several numerical experiments, keeping $R, D_{21}, \mu \equiv$ Consts. The accompanying table (Table 3 ) compares the typical parameters for the slug self-propulsion and its laboratory analogs extracted from the paper of Bico and Quéré [5].

Figure 10 gives some graphical illustrations on this basis. On a careful perusal of Table 3 and Figure 10, respectively, one concludes that the agreement between simulations and experiments is good. As a result, the present study puts forward an important point: an increase in $l_{s}>0$ (by $\delta l_{s}=10$ $[\mathrm{mm}]$, say) weakens the surface tension difference, that is, $\gamma(t, \pi)$. Hence, one concludes that a slug of length $l_{s}$ moves faster than the one whose length slightly differs, for instance, by $\delta l_{s}>0$. Furthermore, perusing Figure 10, we conclude 


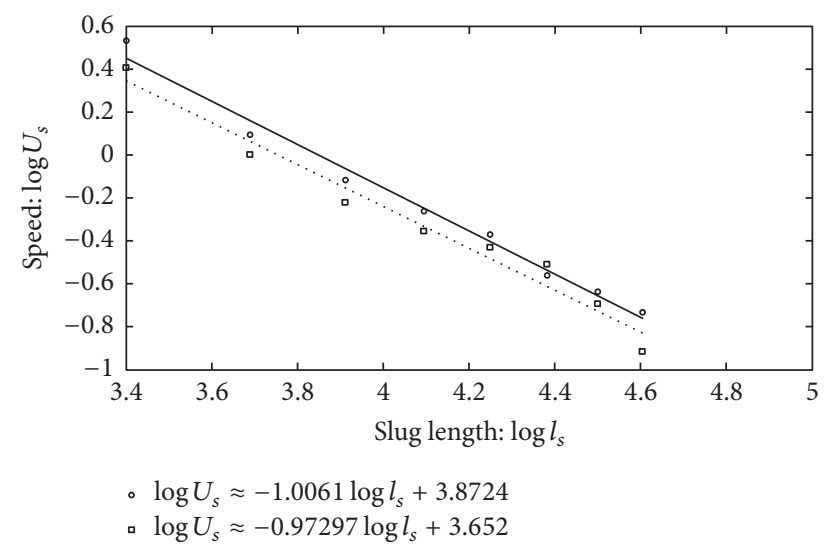

FIGURE 11: Slug velocity versus its length $l_{s}[\mathrm{~mm}], \log U_{s} \approx$ $-1.0061 \log l_{s}+3.8724$. The radius of the capillary tube is $0.4[\mathrm{~mm}]$, and the successive lengths are $l_{1}=16[\mathrm{~mm}]$ and $l_{2}=16.5[\mathrm{~mm}]$. The numerical result (solid line) is compared with that of Bico and Quéré [5] (dotted line). Note that from the graphical result of Bico and Quéré [5] we deduce $\log U_{s} \approx-0.97297 \log l_{s}+3.652$.

TABLE 4: Numerical and experimental magnitudes of the speeds $\left.\left.U_{s}\right\rfloor_{\text {num }}, U_{s}\right\rfloor_{\exp }>0$, respectively, of the slug versus the size $l_{s}>0$ of the slug.

\begin{tabular}{lcccccccc}
\hline \multicolumn{10}{c}{ Results of case study 2.2} \\
\hline$l_{s}[\mathrm{~mm}]$ & 30 & 40 & 50 & 60 & 70 & 80 & 90 & 100 \\
$\left.U_{s}\right\rfloor_{\text {num }}\left[\mathrm{mm} \cdot \mathrm{s}^{-1}\right]$ & 1.7 & 1.1 & 0.89 & 0.77 & 0.69 & 0.57 & 0.53 & 0.48 \\
$\left.U_{s}\right\rfloor_{\exp }\left[\mathrm{mm} \cdot \mathrm{s}^{-1}\right]$ & 1.5 & 1.0 & 0.8 & 0.7 & 0.65 & 0.6 & 0.5 & 0.4 \\
\hline
\end{tabular}

that the dependence of the functional $\chi(\pi)$ restricted to $\left(R, D_{21}, \mu\right)=$ Consts on $l_{s}>0$ evolves on a log-log scale according to

$$
\begin{aligned}
\log \chi(\pi) \approx-\left|K_{l_{s}}\right| \log l_{s}+ & K_{l_{s}, 0}, \\
& \text { where } K_{l_{s}}, K_{l_{s}, 0}=\text { Consts. }
\end{aligned}
$$

As pointed out so far, it is understood that the scalar fields $K_{l_{s}}, K_{l_{s}, 0}$ are partially $\pi$-dependant constants. From Figures 10 and 11 , it may be remarked in passing that

$$
\left.\left.\partial_{t} X_{s}\right\rfloor_{l_{s}=30} \geq \partial_{t} X_{s}\right\rfloor_{l_{s} \geq 30} \text { for given } \pi, t \in(0, T) .
$$

Converting every $\log$-log size-speed $\left(\log l_{s}, \log U_{s}\right)$ pair into the usual $\left(l_{s}, U_{s}\right)$ pair and, further, letting $\left.\left.U_{s}\right\rfloor_{\text {num }}, U_{s}\right\rfloor_{\exp }>$ 0 be the corresponding numerical, experimental values, the tabulation of all these property constants yields Table 4 .

7.3. Case 2.3: Motion of the Slug versus Diffusivity. This section discusses the effects of diffusivity constant $D_{21}>0$. A way of interpreting the latter may be understood on the following basis. At leading order, we deduced from the constitutive equation of $\mathscr{C}^{(l)}(\mathbf{r}, t)$ the following scaling (diffusion length): $l(t) \approx \sqrt{D_{21} t}$, which gave insight of how far the concentration has propagated inside the slug in time $t>0$ for a given diffusivity constant $D_{21}>0$. In Figure 12 is graphed on a log$\log$ scale the speed $U_{s}>0$ of the slug versus the diffusivity

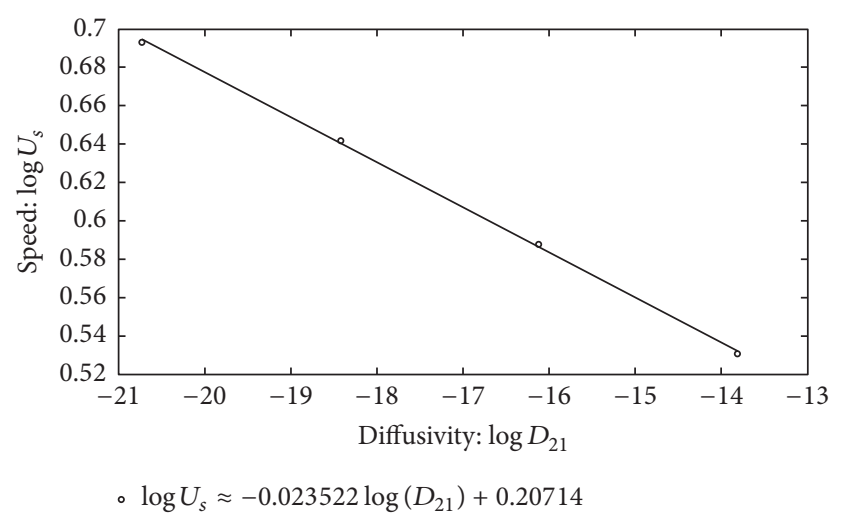

FIGURE 12: Slug velocity versus diffusivity $D_{21}\left[\mathrm{~m}^{2} \cdot \mathrm{s}^{-1}\right]$ in logarithmic scale; solid line is represented by $\log U_{s} \approx-0.023522 \log D_{21}+$ 0.20714 . The radius of the capillary tube is $0.4[\mathrm{~mm}]$, and the successive lengths are $l_{1}=16[\mathrm{~mm}]$ and $l_{2}=16.5[\mathrm{~mm}]$.

TABLE 5: Numerical magnitudes of the speed $\left.U_{s}\right\rfloor_{\text {num }}>0$ of the slug versus the diffusivity $D_{21}>0$ of the slug.

\begin{tabular}{lcccc}
\hline \multicolumn{5}{c}{ Results of case study 2.3} \\
\hline$D_{21}\left[\mathrm{~m}^{2} \cdot \mathrm{s}^{-1}\right]$ & $10^{-6}$ & $10^{-7}$ & $10^{-8}$ & $10^{-9}$ \\
$\left.U_{s}\right\rfloor_{\text {num }}\left[\mathrm{mm} \cdot \mathrm{s}^{-1}\right]$ & 1.7 & 1.8 & 1.9 & 2.0 \\
\hline
\end{tabular}

constant $D_{21}>0$. When every log-log diffusivity-speed $\left(\log D_{21}, \log U_{s}\right)$ pair is converted into the usual $\left(D_{21}, U_{s}\right)$ pair and, further, $\left.U_{s}=U_{s}\right\rfloor_{\text {num }}>0$ to stress that the dependence of the speed of the slug on the diffusivity has not been studied numerically, the tabulation of these property constants yields Table 5.

As a result, incrementing the diffusivity constant $D_{21}>0$ by $\delta D_{21}>0$ yields

$$
\left.\gamma(t, \pi)\rfloor_{D_{21}+\delta D_{21}}<\gamma(t, \pi)\right\rfloor_{D_{21}}
$$

for given $\pi, t \in(0, T)$.

On the whole, augmenting $D_{21}>0$ therefore compels the propulsion force, which is proportional to the surface tension difference $\gamma(t, \pi)$, to decrease in magnitude. As a consequence, the transport coefficient $D_{21}>0$ therefore does have bearing on the motion of the slug.

In other words, augmenting the magnitude of the diffusivity constant $D_{21}>0$ results in not only diminishing the magnitude of the slug velocity $U_{s}>0$, but also causing the propulsion force to vanish/die earlier. As shown in Figure 12, one clearly sees that the $\pi$-dependent functional $\chi(\boldsymbol{\pi})$ now restricted to $\left(R, l_{s}, \mu\right)=$ Consts evolves logarithmically with the diffusion constant $D_{21}>0$ according to the following loglog relationship:

$$
\begin{aligned}
\log \chi(\boldsymbol{\pi}) \approx-\left|K_{D_{21}}\right| & \log D_{21}+K_{D_{21}, 0} \\
& \text { where } K_{D_{21}}, K_{D_{21}, 0}=\text { Consts. }
\end{aligned}
$$

As pointed out so far, it is understood that the scalar fields $K_{D_{21}}, K_{D_{21}, 0}$ are partially $\pi$-dependant constants. This is an 


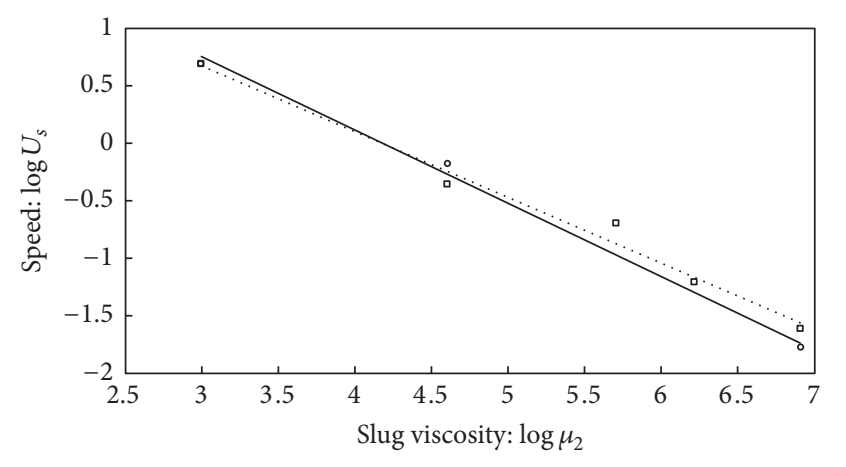

- $\log U_{s} \approx-0.63452 \log \mu_{2}+2.6509$

- $\log U_{s} \approx-0.57214 \log \mu_{2}+2.39$

FIGURE 13: Slug velocity versus viscosity $\mu_{2}[\mathrm{mPa} \cdot \mathrm{s}]$ in logarithmic scale; numerical result, approximated by $\log U_{s} \approx-0.63452 \log \mu_{2}+$ 2.6509 , is graphed in solid line; experimental result extracted from earlier work approximated by $\log U_{s} \approx-0.57214 \log \mu_{2}+2.39$ is in dotted line. The radius of the capillary tube is $0.51[\mathrm{~mm}]$, and the successive lengths are $l_{1}=16[\mathrm{~mm}]$ and $l_{2}=16.5[\mathrm{~mm}]$; also, the viscosity $\mu_{1}=17[\mathrm{mPa} \cdot \mathrm{s}]$ is kept constant.

important feature which has not been considered in any of the two papers of Bico and Quéré $[1,5]$. It may be remarked further that (39) tends to prove that the logarithm of the speed of the slug $\log U_{s}$ (since $\chi(\pi) \equiv U_{s}$ ) is proportional to the logarithm of the diffusivity constant $\log D_{21}$. Moreover, since $l(t) \approx \sqrt{D_{21} t}$ (that diffusion length which provides a measure of how far the chemical concentration has propagated into the slug in the $z$-direction by diffusion in time $t>0$ ), the existence of a reference time $\tau_{\text {ref }}>0$ can be assumed such that

$$
\begin{aligned}
\operatorname{Ord}\left(\log U_{s}\right) & \sim \operatorname{Ord}\left(\log \left(\frac{d l}{d t}\right)\right) \\
& \sim \log \left(\frac{D_{21}}{\tau_{\text {ref }}}\right)+\text { Const. }
\end{aligned}
$$

from which the shape of (39) at once comes to light. Physically speaking, if the diffusion constant $D_{21}>0$ is to furnish us with a means of strengthening the self-propulsion mechanism, it is evident that it should be low in magnitude. Hence, this establishes a relation between the diffusion constant $D_{21}>0$ and the speed $U_{s}>0$ of the slug. The next section considers the speed $U_{s}>0$ of the slug versus the viscosity constant $\mu>0$.

7.4. Case 2.4: Motion of Slug versus Viscosity. The nature of the movement of the slug with the character of the viscous force is here considered by establishing a relationship relating the speed $U_{s}>0$ of the slug to the viscosity constant $\mu>0$. To effect this purpose we ran several simulations, changing the magnitude of the property $\mu>0$ at each run while restricting other property constants to those values appearing in Table 1. Further, at each run the pair of scalar fields $(\mu, \chi(\pi))$ is noted. In Figures 13 and 14 are plotted the pairs of scalar fields $\left(\mu_{i}, \chi(\pi)\right)_{i=1,2}$ on a log-log scale. As one can see from Figures 13 and 14 , the dependence of the functional $\chi(\pi)$ finally

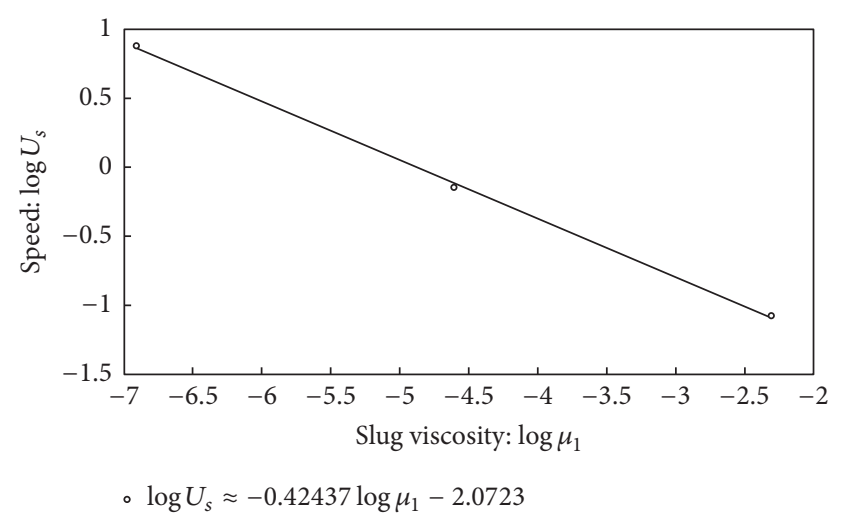

FIGURE 14: Slug velocity versus viscosity $\mu_{1}$ [mPa.s] in logarithmic scale; numerical result, approximated by $\log U_{s} \approx-0.42437 \log \mu_{1}-$ 2.0723 , is graphed in solid line. The radius of the capillary tube is $0.51[\mathrm{~mm}]$, and the successive lengths are $l_{1}=16[\mathrm{~mm}]$ and $l_{2}=16.5$ [mm]; also, the viscosity $\mu_{2}=17[\mathrm{mPa} \cdot \mathrm{s}]$ is kept constant.

restricted to $\left(R, l_{s}, D_{21}\right) \equiv$ Consts on $\mu_{i}>0$ (with $i=1$, 2) evolves on a log-log scale according to

$$
\begin{aligned}
\log \chi(\pi) \approx-\left|K_{\mu}\right| \log \mu+ & K_{\mu, 0} \\
& \text { where } K_{\mu}, K_{\mu, 0}=\text { Consts. }
\end{aligned}
$$

As pointed out so far, it is understood that the scalar fields $K_{\mu}, K_{\mu, 0}$ are partially $\pi$-dependant constants. Moreover, because this expression of $U_{s}>0$ in terms of $D_{21}>0$ is algebraically similar to that established in the foregoing section, our thoughts are thereby led to regard a decrease in $\mu>0$ resulting in an exponential increase in $U_{s}>0$, which manifests the nature of viscous effect.

To the informed reader the law $U_{s} \propto \mu^{-1}$ is obvious whenever the Poiseuille law is pronounced in the mathematical development but, to the uninformed reader, the law $U_{s} \propto \mu^{-1 / 2}$ is unobvious. Thus, before advancing further, it might be interesting to justify this point.

Since, in the earlier section (see Section 7.2), we showed that $U_{s} \propto l_{s}^{-1}$ and, from Poiseuille flow, we have $U_{s} \propto \mu^{-1}$, it is plain that

$$
\begin{aligned}
U_{s} & \propto \frac{1}{\mu_{1}^{\alpha} \cdot \mu_{2}^{1-\alpha}} \quad\left(\text { if } \mu=\mu_{1}, \mu_{1}=\mu_{2} ; \alpha \in(0,1)\right) \\
& \propto \frac{1}{\mu_{1}^{\alpha}} \quad\left(\text { if } \mu_{2}=\text { Const.; } \alpha \in(0,1)\right) \\
& \propto \frac{1}{\mu_{2}^{1-\alpha}} \quad\left(\text { if } \mu_{1}=\text { Const.; } \alpha \in(0,1)\right) .
\end{aligned}
$$

Equation (42) states that the dependence of the slug speed on the dynamic viscosity $\mu_{i}>0$ of the $i$-fluid (with $i=1,2$ ) is such that $U_{s} \propto \mu_{i}^{-\beta}$ for some real $\beta \in(0,1)$. Moreover, it also states that if such dependence is known for one fluid, the other can be deduced, since whenever $U_{s} \propto \mu_{1}^{-\beta}$ necessarily $U_{s} \propto \mu_{2}^{1-\beta}$. Otherwise the condition $U_{s} \propto \mu^{-1}$ will be violated. Taking advantage of the properties of logarithms 
to convert the second and third lines of (42) into linear relationships, one obtains

$$
\begin{aligned}
& \log U_{s}=\kappa_{i} \cdot \log \mu_{i}+\text { Const. } \\
& \qquad \forall i \in\{1,2\}, \text { where } \sum_{i=1,2} \kappa_{i}=1 .
\end{aligned}
$$

Through numerical experiments, we deduced $\left(\kappa_{1}, \kappa_{2}\right)=$ $(-0.42437,-0.63452)$. Since $\sum_{i=1,2} \kappa_{i}=1.05889$, it follows that $\sum_{i=1,2} \kappa_{i} \approx 1$, hence satisfying (43) and, therefore, (42). We have thus justified the results appearing in Figures 13 and 14.

Having studied the motion of the slug under variations of the property vector $\pi=\left(R, l_{s}, D_{21}, \mu\right)^{T}$, we propose in the next section an algebraic expression, characteristics of the nature of the movement of the slug, on this ground.

\section{Power-Law Slug Dynamics}

In the foregoing sections, we examined the response of the slug subjected to the property constant $\pi$. We also illustrated through case by case studies the effects of the latter on the movement of the slug. This section aims at proposing a power-law model, which might well be termed the Law of Movement of the Slug. If we recall attention to the foregoing sections as regards the nature of the functional $\chi(\pi)$ with respect to the property $\pi$, we shall see that the speed $U_{s}>0$ of the slug is related to the elements of the property vector $\pi=\left(R, l_{s}, D_{21}, \mu\right)^{T}$ as

$$
\begin{aligned}
& U_{s} \\
& \approx \begin{cases}\left|K_{R}\right| R+K_{R, 0}, & K_{R}, K_{R, 0}=\text { Consts; see }(35) ; \\
-\left|K_{l_{s}}\right| \log l_{s}+K_{l_{s}, 0}, & K_{l_{s}}, K_{l_{s}, 0}=\text { Consts; see }(36) ; \\
-\left|K_{D_{21}}\right| \log D_{21}+K_{D_{21}, 0} & K_{D_{21}}, K_{D_{21}, 0}=\text { Consts; see }(39) ; \\
-\left|K_{\mu}\right| \log \mu+K_{\mu, 0} & K_{\mu}, K_{\mu, 0}=\text { Consts; see (41). }\end{cases}
\end{aligned}
$$

On the other hand, from the above graphical results it is observed that the quantities $\left|K_{R}\right|,\left|K_{l_{s}}\right|, \mid K$, and $\left|K_{\mu}\right|$, respectively, roughly interrelate themselves as follows:

$$
\begin{aligned}
\operatorname{Ord}\left(\left|K_{R}\right|\right) & \sim \operatorname{Ord}\left(\left|K_{l_{s}}\right|\right) \sim \operatorname{Ord}\left(2\left|K_{\mu}\right|\right) \\
& \sim \operatorname{Ord}\left(50\left|K_{D_{21}}\right|\right) \quad(\sim 1) .
\end{aligned}
$$

If we pause ourselves for a moment and ponder on the above relationship, we would obviously conclude that the relative effect arising from the diffusion constant $D_{21}>0$ is little. Thence, paralleling (31) with that which one would deduce at leading order in a fully developed slug flow in a circular pipe of the same radius $R$ together with the neglect of $\log D_{21}$ yields

$$
\begin{gathered}
U_{s} \sim \xi\left(\frac{R\left(\gamma_{1,0}-\gamma_{2,0}\right)}{4 \mu l_{s}}\right)^{|\alpha|}, \\
U_{\mathrm{eff}} \sim \xi\left(\frac{R\left(\gamma_{1,0}-\gamma_{2,0}\right)}{4 \mu l_{s}}\right)^{|\alpha|-1},
\end{gathered}
$$

where $\alpha=K_{l_{s}} \sim K_{\mu}>0$ and $\xi>0$. Writing the speed $U_{s}>0$ of the slug as such puts forward the term of the concept of a Poiseuille flow. In these, the scalar fields $\xi>0,|\alpha|$ define the slug dynamics consistency and slug behaviour indices (with $|\alpha| \approx 1.0$ ); the scalar field $U_{\text {eff }}>0$ is an effective slug velocity. To deduce the scalar field $\xi>0$ on this basis one may then proceed through practical experimentation.

In the paper of Lunati and Or [35], the authors mentioned that the slug velocity, for the case of a gravity-driven flow in a capillary tube, is lower (without any precise order of magnitude) than deduced in a Poiseuille flow. Here, the result points to a contrary conclusion, for using the data given in Table 1 one demonstrates that the speed $U_{s}>0$ of the slug is greater by an $\epsilon$-order $\epsilon \sim 10^{-2}$, roughly. Such discrepancy might be due to the neglect of dissipative effects occurring at the wedges of the slug.

To this end, it seems therefore plausible to assume the following general description:

$$
\begin{aligned}
& X_{s}(t, \pi) \\
& \approx X_{s, \infty}\left(1-\exp \left\{-\frac{\xi\left(t-t_{0}\right)}{X_{s, \infty}}\left(\frac{R\left(\gamma_{1,0}-\gamma_{2,0}\right)}{4 \mu l_{s}}\right)^{|\alpha|}\right\}\right) .
\end{aligned}
$$

We agree here that the property $\pi$ includes all property constants appearing on the right-hand of (47), as can be easily understood. There is an immediate consequence which follows from (47). Setting $\lambda=R\left(\gamma_{1,0}-\gamma_{2,0}\right) / 4 \mu l_{s}$, one concludes that $\partial_{t} X_{s} \propto \lambda$ in the early-time (linear) regime, whereas $\partial_{t} X_{s} \propto \exp (-\lambda)$ in the late-time (nonlinear) regime. Since a Poiseuille flow is one such that its flow fields satisfy $\|\mathbf{u}\| \propto \lambda$, it follows that the slug flow obeys Poiseuille flow only in the early-time regime.

To round off the present discussion, we point out that, with $\xi\left(l_{s}=30[\mathrm{~mm}]\right) \approx 22.3633$ and $\xi\left(l_{s}=60[\mathrm{~mm}]\right) \approx$ 13.2664 along with the numerical data given in Tables 2 and 3 , plotting of (47) coincides with those given (in solid lines) in Figure 15, for comparing (29) with (47) gives

$$
\xi(\pi) \approx \eta_{1} \eta_{2}\left(\frac{R\left(\gamma_{1,0}-\gamma_{2,0}\right)}{4 \mu l_{s}}\right)^{1-|\alpha|} \approx 1
$$

$$
\text { for given } \pi \text {, since }|\alpha| \approx 1 \text {. }
$$

Finally, combining (23) with (47) and (48), one arrives at

$$
\begin{aligned}
& \partial_{t} X_{s} \approx U_{s}\left(1-\frac{X_{s}}{X_{s, \infty}}\right) \leq U_{s}, \\
& \frac{d X_{s}}{d \gamma} \approx U_{\text {eff }}\left(1-\frac{X_{s}}{X_{s, \infty}}\right) \leq U_{\text {eff }}
\end{aligned}
$$

Hence, it would appear that (49) hold for every fixed instant of time $t>0$, provided that $\partial_{t} \gamma \approx$ Const. Hence, if we let the scalar field $U_{p}>0$ be the Poiseuille velocity field, one deduces

$$
\partial_{t} X_{s} \approx \frac{R\left(\gamma_{1,0}-\gamma_{2,0}\right)}{4 \mu l_{s}} \frac{d X_{s}}{d \gamma} \approx U_{p} \frac{d X_{s}}{d \gamma}
$$




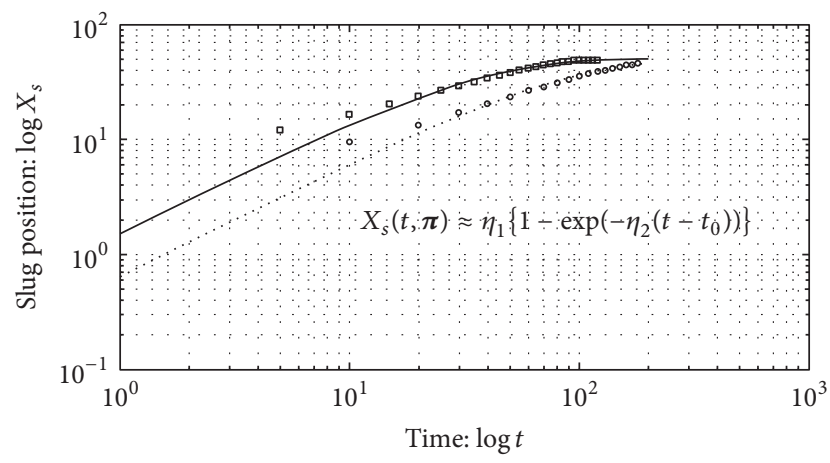

Figure 15: Results with (47) and numerical data. Equation (47) is described in solid line for $l_{s}=30[\mathrm{~mm}]$ and in dotted line for $l_{s}=60$ $[\mathrm{mm}]$. Likewise, numerical data is described with circles for $l_{s}=30$ $[\mathrm{mm}]$ and with squares for $l_{s}=60[\mathrm{~mm}]$.

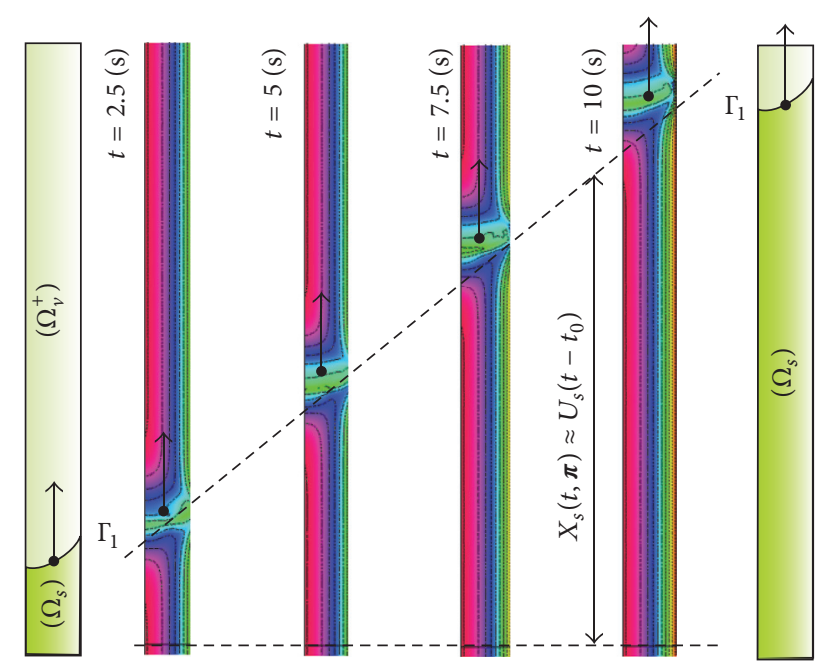

FIGURE 16: Illustration of the flow contours at the sequence of times $\left(t_{k}\right)_{k \in[1,5]}=(5 k / 2)_{k \in[0,4]}[s]$.

since $U_{p} \approx U_{s} \cdot U_{\mathrm{eff}}^{-1}$. As pointed out before, it then follows that $\operatorname{Ord}\left(d X_{s} / d \gamma\right) \sim \epsilon^{-2}$.

In Figure 16 are displayed the flow contours generated by the movement of the slug into its capillary tube for $t=5 k / 2$, where $k \in[1,4]$, the time evolution of the advancing interface in the early-time regime. The region $\Omega_{s}=\Omega_{1} \cup \Omega_{2}$ designates the domain partially filled with the miscible fluid; the region $\Omega_{v}^{+}$designates that occupied by the vapour phase fluid.

In Figure 17 are displayed the distribution of the fraction volume of fluids $\phi_{12}(\mathbf{r}, t)$ within the regions $\Omega_{1} \cup \Omega_{2}$ and $\Omega_{v}^{-} \cup$ $\Omega_{v}^{+}$, the time evolution of the advancing interface in the earlytime regime, for the sequence of time $t_{k}=2 k$, where $k \in$ $[0,5]$. This completes the discussion of this part.

\section{Conclusion}

Hitherto, it is believed insofar as we are knowledgeable that this is the first time that such a study has been undertaken. Moreover, perusing our results, one admittedly concludes

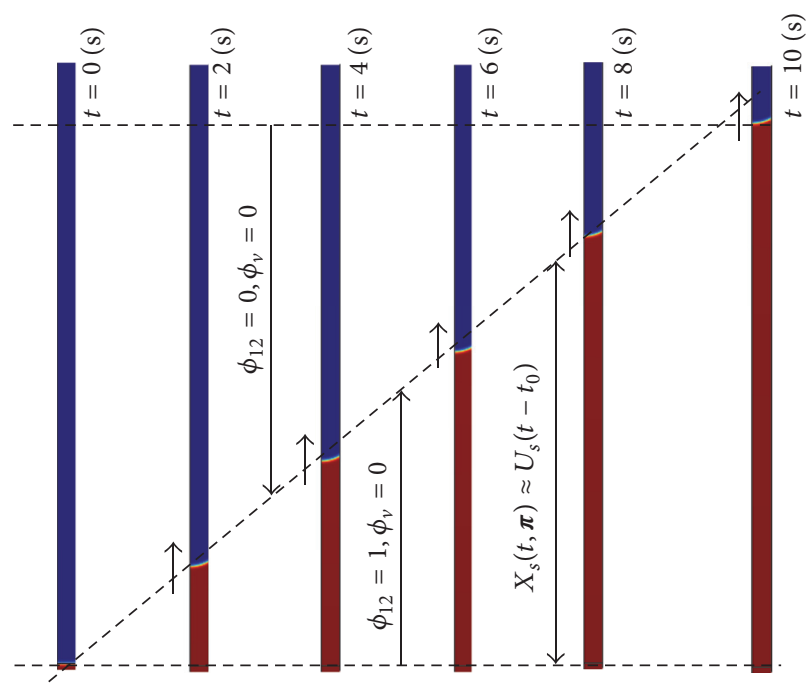

FIGURE 17: Evolution of the fraction volume of fluid to illustrate the front interface $\Gamma_{1}$ of the slug at the sequence of times $\left(t_{k}\right)_{k \in[1,5]}=$ $(2 k)_{k \in[0,5]}[\mathrm{s}]$.

that ours are very similar to the observations reported by Bico and Quéré $[1,5]$.

On the whole, we have observed the following points.

(i) A mathematical model is developed and implemented in COMSOL Multiphysics.

(ii) Two regimes were identified: the early-time (linear) and late-time (nonlinear) regimes. In the early-time regime, the speed of the slug is proportional to the Poiseuille flow, whereas in the late-time regime it is proportional to the exponential of the Poiseuille flow.

(iii) Exploring the linear regime parametrically, the dependence of the speed of the slug on its size $l_{s}$, diffusivity $D_{21}$ and dynamic viscosity $\mu$ constants, and the radius $R$ of the capillary tube is found to be powerlaw functions. Moreover, the speed is found to be insensitive to $D_{21}$. These parametric studies show that the size and properties of the slug do have bearing on its motion.

(iv) For an initially chosen set of physical properties, the position of the slug evolves exponentially with respect to time according to (47).

Of the above statements, the second one confirms the conclusion reached by Piroird et al. [43] in one of their papers, namely, that on a long time scale two regimes were observed; and the third one confirms that found in another paper of Piroird et al. [44], namely, that by varying the geometrical properties associated with the droplet and its capillary tube, the nature of the flow changes.

Additionally, it should be stressed that it is not necessary to argue or even to enumerate in the context of practical applications, for we believe that the above mathematical development is sufficient to prove its usefulness in practical applications. However, it should be borne in mind that, without the numerical data $\xi,|\alpha|$, and $X_{s, \infty}$, the proposed 
model is insufficient to allow one to predict the position of the slug at some time $t_{0} \geq 0$ thereafter. Nonetheless, it does indicate that in this range it is not possible to describe the dynamics of the slug without knowing, for instance, at what time, $t_{\infty}>0$ say, the slug must reach its destination, $X_{s, \infty}>0$ say. Such is the conclusion to which we are led.

Taking into account the foregoing discussions, it may be declared that our model, namely, (47), can do good service in the selection of microfluidic (or more precisely capillary) devices. To conclude, it may be said that the outcome of the present part results in the establishment of the following proposition.

Proposition 1. Given $\left(\kappa_{1}, \kappa_{2}\right)$, there is a pair of $\pi$-dependent property constants. By a proper choice of the property $\pi=$ $\left(\pi_{1}, \ldots, \pi_{d}\right) \in \mathbb{R}^{d}$, the path of a self-propelling slug in a capillary tube obeys the following law:

$$
\begin{aligned}
& X(\boldsymbol{\pi} ; t)=\kappa_{1}\left\{1-\exp \left(-\kappa_{2} t\right)\right\} \\
& \text { with } \kappa_{i}=f_{i}(\pi), \text { for } i=12 .
\end{aligned}
$$

We name (51) the Prototype Slug Self-Propulsion Model Equation because it gave agreement to both time and physical properties of the slug.

\section{Competing Interests}

The authors declare that they have no competing interests.

\section{References}

[1] J. Bico and D. Quéré, "Self-propelling slugs," Journal of Fluid Mechanics, vol. 467, pp. 101-127, 2002.

[2] J. Thomson, "On certain curious motions observable at the surfaces of wine and other alcoholic liquors," Philosophical Magazine, vol. 10, pp. 330-333, 1855.

[3] C. Marangoni, On the Expansion of Liquid Floating on the Surface of Another Liquid, Tipographia dei Fratelli Fusi, Pavia, Italy, 1865.

[4] L. E. Scriven and C. V. Sternling, "The marangoni effects," Nature, vol. 187, no. 4733, pp. 186-188, 1960.

[5] J. Bico and D. Quéré, "Liquid trains in a tube," Europhysics Letters, vol. 51, no. 5, pp. 546-550, 2000.

[6] D. K. N. Sinz and A. A. Darhuber, "Self-propelling surfactant droplets in chemically-confined microfluidics-Cargo transport, drop-splitting and trajectory control," Lab on a ChipMiniaturisation for Chemistry and Biology, vol. 12, no. 4, pp. 705-707, 2012.

[7] H. Bouasse, Capillarit, Phnomnes Superficiel, Delagrave, Paris, France, 1924.

[8] M. M. Weislogel, "Steady spontaneous capillary flow in partially coated tubes," AIChE Journal, vol. 43, no. 3, pp. 645-654, 1997.

[9] F. D. Dos Santos and T. Ondarçuhu, "Free-running droplets," Physical Review Letters, vol. 75, no. 16, pp. 2972-2975, 1995.

[10] P. G. De Gennes, “The dynamics of reactive wetting on solid surfaces," Physica A: Statistical Mechanics and Its Applications, vol. 249, no. 1-4, pp. 196-205, 1998.

[11] F. Hauksbee, "An account of an experiment touching the direction of oil of oranges, between two glass planes, towards any side of them that is nearest press'd together," Philosophical Transactions, vol. 27, pp. 395-396, 1712.

[12] S. K. Cho and H. Moon, "Electrowetting on dielectric (EWOD): new tool for bio/micro fluids handling," Biochip Journal, vol. 2, no. 2, pp. 79-96, 2009.

[13] L. Y. Yeo and H.-C. Chang, "Static and spontaneous electrowetting," Modern Physics Letters B, vol. 19, no. 12, pp. 549-569, 2005.

[14] T. B. Jones, K.-L. Wang, and D.-J. Yao, "Frequency-dependent electromechanics of aqueous liquids: electrowetting and dielectrophoresis," Langmuir, vol. 20, no. 7, pp. 2813-2818, 2004.

[15] S. K. Cho, H. Moon, and C.-J. Kim, "Creating, transporting, cutting, and merging liquid droplets by electrowetting-based actuation for digital microfluidic circuits," Journal of Microelectromechanical Systems, vol. 12, no. 1, pp. 70-80, 2003.

[16] M. G. Pollack, R. B. Fair, and A. D. Shenderov, "Electrowettingbased actuation of liquid droplets for microfluidic applications," Applied Physics Letters, vol. 77, no. 11, pp. 1725-1726, 2000.

[17] J. Lee and C.-J. Kim, "Surface-tension-driven microactuation based on continuous electrowetting," Journal of Microelectromechanical Systems, vol. 9, no. 2, pp. 171-180, 2000.

[18] T. B. Jones, M. Gunji, M. Washizu, and M. J. Feldman, "Dielectrophoretic liquid actuation and nanodroplet formation," Journal of Applied Physics, vol. 89, no. 2, pp. 1441-1448, 2001.

[19] M. L. Ford and A. Nadim, "Thermocapillary migration of an attached drop on a solid surface," Physics of Fluids, vol. 6, no. 9, pp. 3183-3185, 1994.

[20] M. K. Smith, “Thermocapillary migration of a two-dimensional liquid droplet on a solid surface," Journal of Fluid Mechanics, vol. 294, pp. 209-230, 1995.

[21] A. A. Darhuber, J. M. Davis, S. M. Troian, and W. W. Reisner, "Thermocapillary actuation of liquid flow on chemically patterned surfaces," Physics of Fluids, vol. 15, no. 5, pp. 1295-1304, 2003.

[22] A. A. Darhuber, J. P. Valentino, S. M. Troian, and S. Wagner, "Thermocapillary actuation of droplets on chemically patterned surfaces by programmable microheater arrays," Journal of Microelectromechanical Systems, vol. 12, no. 6, pp. 873-879, 2003.

[23] A. Renaudin, P. Tabourier, V. Zhang, J. C. Camart, and C. Druon, "SAW nanopump for handling droplets in view of biological applications," Sensors and Actuators B: Chemical, vol. 113, no. 1, pp. 389-397, 2006.

[24] L. Y. Yeo and J. R. Friend, "Ultrafast microfluidics using surface acoustic waves," Biomicrofluidics, vol. 3, no. 1, Article ID 012002, 2009.

[25] J. Pfahler, J. Harley, H. Bau, and J. Zemel, "Liquid transport in micron and submicron channels," Sensors and Actuators A, vol. 22, no. 1-3, pp. 431-434, 1989.

[26] P. G. De Gennes, "Wetting: statics and dynamics," Reviews of Modern Physics, vol. 57, no. 3, pp. 827-863, 1985.

[27] P. G. De Gennes, "Eponges filantes," Comptes Rendus de l'Académie des Sciences, vol. 323, pp. 663-667, 1996.

[28] M. E. R. Shanahan and P. G. De Gennes, "The motion of long bubbles in tubes," Comptes Rendus de l'Académie des Sciences (Paris) IIb, vol. 324, p. 261, 1997.

[29] M. Sellier, V. Nock, and C. Verdier, "Self-propelling, coalescing droplets," International Journal of Multiphase Flow, vol. 37, no. 5, pp. 462-468, 2011.

[30] M. Sellier, V. Nock, C. Gaubert, and C. Verdier, "Droplet actuation induced by coalescence: experimental evidences and 
phenomenological modeling," European Physical Journal: Special Topics, vol. 219, no. 1, pp. 131-141, 2013.

[31] S. Karpitschka and H. Riegler, "Quantitative experimental study on the transition between fast and delayed coalescence of sessile droplets with different but completely miscible liquids," Langmuir, vol. 26, no. 14, pp. 11823-11829, 2010.

[32] S. Karpitschka and H. Riegler, "Noncoalescence of sessile drops from different but miscible liquids: hydrodynamic analysis of the twin drop contour as a self-stabilizing traveling wave," Physical Review Letters, vol. 109, no. 6, Article ID 066103, 5 pages, 2012.

[33] A. A. Darhuber, J. P. Valentino, J. M. Davis, S. M. Troian, and S. Wagner, "Microfluidic actuation by modulation of surface stresses," Applied Physics Letters, vol. 82, no. 4, pp. 657-659, 2003.

[34] A. A. Darhuber, J. P. Valentino, J. M. Davis, and S. M. Troian, "Principles of microuidic actuation by modulation of surface stresses," Annual Review of Fluid Mechanics, vol. 37, pp. 425-455, 2005.

[35] I. Lunati and D. Or, "Gravity-driven slug motion in capillary tubes," Physics of Fluids, vol. 21, no. 5, Article ID 052003, 2009.

[36] J. Bico and D. Quéré, "Falling slugs," Journal of Colloid and Interface Science, vol. 243, no. 1, pp. 262-264, 2001.

[37] P. Carles and A. M. Cazabat, "Spreading involving the marangoni effect: some preliminary results," Colloids and Surfaces, vol. 41, pp. 97-105, 1989.

[38] P. Carles and A. M. Cazabat, "Spreading of oil drops under a solvent vapor: inuence of marangoni effect," Progress in Colloid and Polymer Science, vol. 82, pp. 76-81, 1990.

[39] P. Yue, C. Zhou, and J. J. Feng, "Sharp-interface limit of the Cahn-Hilliard model for moving contact lines," Journal of Fluid Mechanics, vol. 645, pp. 279-294, 2010.

[40] D. Jacqmin, "Calculation of two-phase Navier-Stokes flows using phase-field modeling," Journal of Computational Physics, vol. 155, no. 1, pp. 96-127, 1999.

[41] O. K. Matar and R. V. Craster, "Models for Marangoni drying," Physics of Fluids, vol. 13, no. 7, pp. 1869-1883, 2001.

[42] S. B. G. M. O’Brien, “On Marangoni drying: nonlinear kinematic waves in a thin film," Journal of Fluid Mechanics, vol. 254, pp. 649-670, 1993.

[43] K. Piroird, C. Clanet, and D. Quéré, "Detergency in a tube," Soft Matter, vol. 7, no. 16, pp. 7498-7503, 2011.

[44] K. Piroird, C. Clanet, and D. Queźreź, "Capillary extraction," Langmuir, vol. 27, no. 15, pp. 9396-9402, 2011. 


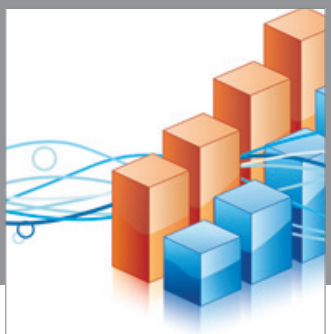

Advances in

Operations Research

vatem alat4

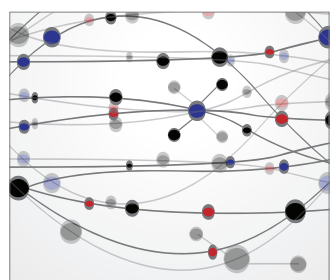

\section{The Scientific} World Journal
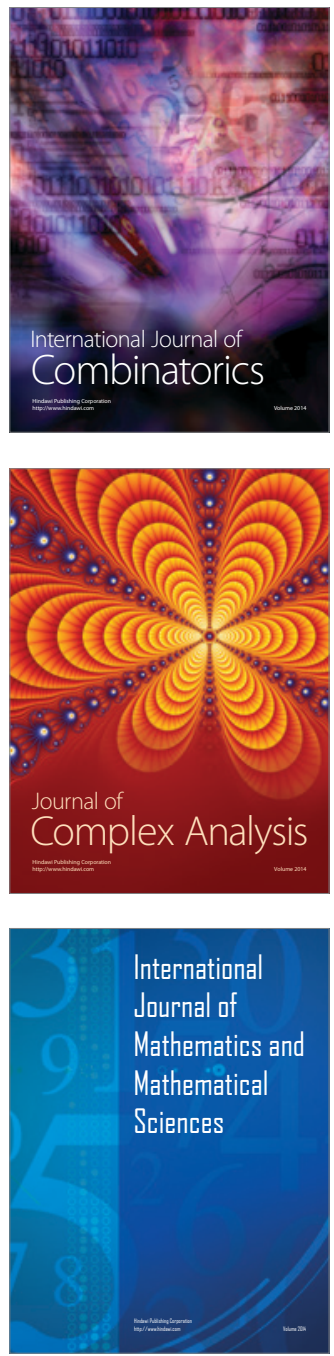
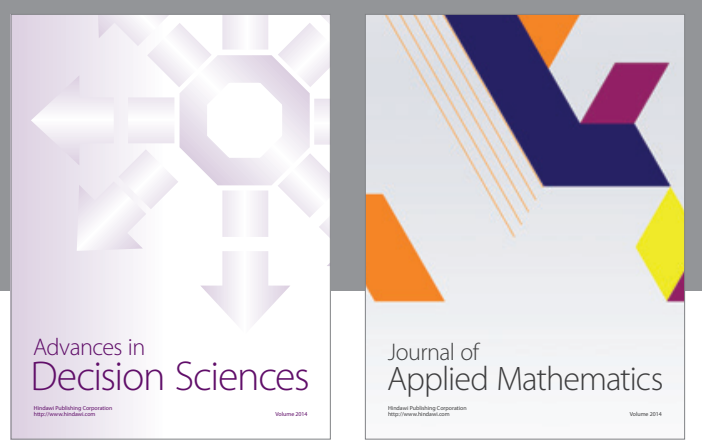

Algebra

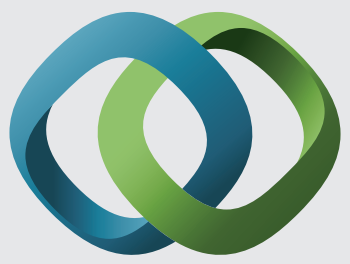

\section{Hindawi}

Submit your manuscripts at

http://www.hindawi.com
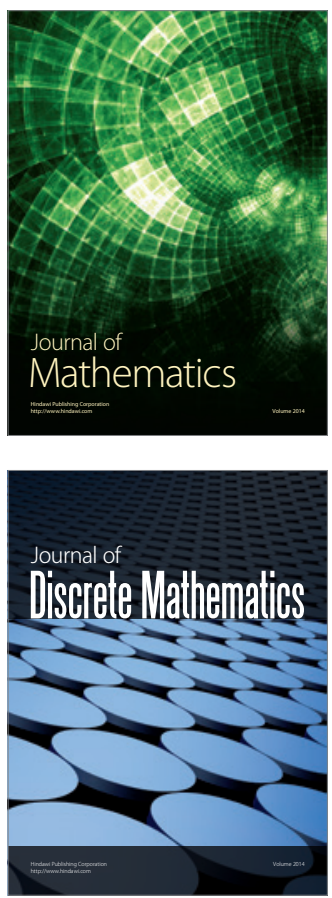

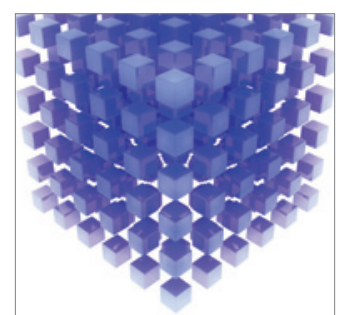

Mathematical Problems in Engineering
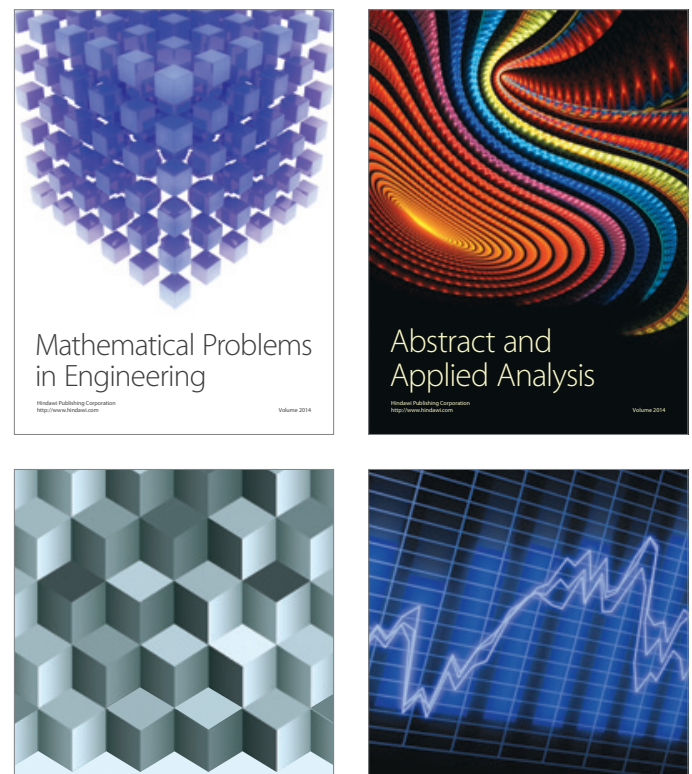

Journal of

Function Spaces

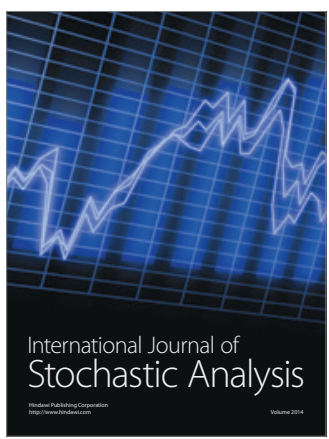

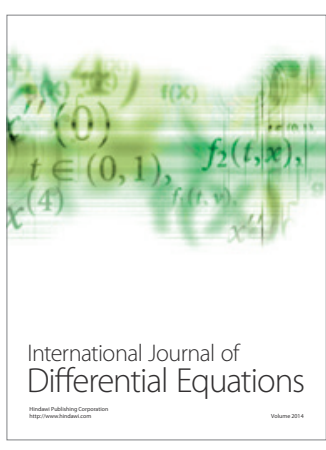
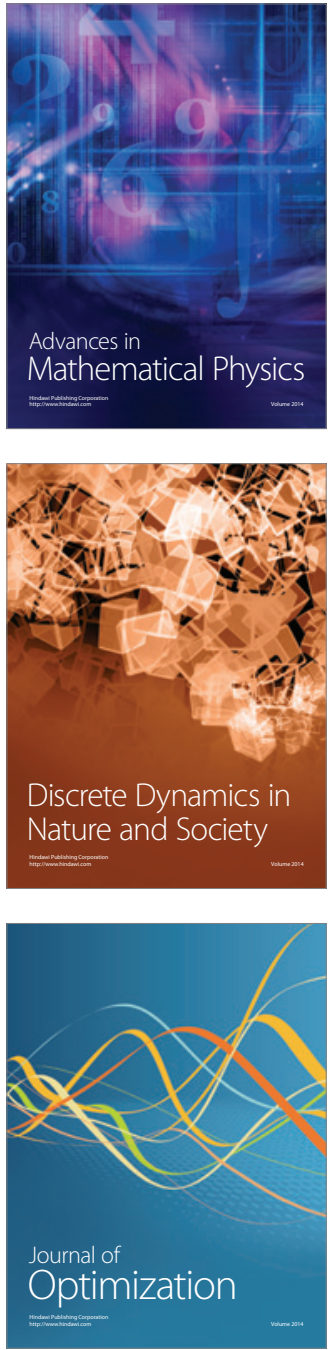